\title{
Isolation and Total Synthesis of Kinenzoline,
} an Antitrypanosomal Linear Depsipeptide Isolated from a Marine Salileptolyngbya sp.

\section{Cyanobacterium}

Naoaki Kurisawa,$\uparrow$ Keisuke Otomo, $\uparrow$ Arihiro Iwasaki, $\uparrow$ Ghulam Jeelani, Tomoyoshi Nozaki, $¥$ Kiyotake Suenaga* $\dagger$

$\dagger$ Department of Chemistry, Faculty of Science and Technology, Keio University, 3-14-1 Hiyoshi, Kohoku-ku, Yokohama, Kanagawa 223-8522, Japan

$\$$ Department of Biomedical Chemistry, Graduate School of Medicine, The University of Tokyo, 7-3-1 Hongo, Bunkyo-ku, Tokyo 113-0033, Japan

*Corresponding Author's E-mail Address: suenaga@chem.keio.ac.jp

Contents

Figure S1. ${ }^{1} \mathrm{H}$ NMR (400 MHz, $\left.\mathrm{CD}_{3} \mathrm{OD}\right)$ spectrum of kinenzoline (1)

Figure $\mathrm{S} 2 .{ }^{13} \mathrm{C}\{1 \mathrm{H}\} \mathrm{NMR}\left(100 \mathrm{MHz}, \mathrm{CD}_{3} \mathrm{OD}\right)$ spectrum of kinenzoline (1) S3

Figure S3. COSY (400 MHz, $\left.\mathrm{CD}_{3} \mathrm{OD}\right)$ spectrum of kinenzoline (1) S4

Figure S4. $\mathrm{HMQC}\left(400 \mathrm{MHz}, \mathrm{CD}_{3} \mathrm{OD}\right)$ spectrum of kinenzoline (1) S5

Figure S5. $\mathrm{HMBC}\left(400 \mathrm{MHz}, \mathrm{CD}_{3} \mathrm{OD}\right)$ spectrum of kinenzoline (1) S6

Figure S6. NOESY (400 MHz, $\left.\mathrm{CD}_{3} \mathrm{OD}\right)$ spectrum of kinenzoline (1) S7

Figure S7. Determination of the absolute configuration of kinenzoline (1) S8-11

$\begin{array}{lr}\text { Figure S8. Phylogenic tree of the cyanobacterium } & \text { S12 }\end{array}$

Figure S9. ${ }^{1} \mathrm{H}$ NMR ( $\left.400 \mathrm{MHz}, \mathrm{CDCl}_{3}\right)$ spectrum of $2 \quad \mathrm{~S} 13$

Figure $\mathrm{S} 10 .{ }^{1} \mathrm{H}$ NMR (400 MHz, $\left.\mathrm{CD}_{3} \mathrm{OD}\right)$ spectrum of $4 \quad \mathrm{~S} 14$

Figure S11. ${ }^{1} \mathrm{H}$ NMR $\left(400 \mathrm{MHz}, \mathrm{CD}_{3} \mathrm{OD}\right)$ spectrum of $5 \quad \mathrm{~S} 15$

Figure $\mathrm{S} 12 .{ }^{1} \mathrm{H} \mathrm{NMR}\left(400 \mathrm{MHz}, \mathrm{CDCl}_{3}\right)$ spectrum of $7 \quad \mathrm{~S} 16$

Figure S13. ${ }^{1 \mathrm{H}} \mathrm{NMR}\left(400 \mathrm{MHz}, \mathrm{CD}_{3} \mathrm{OD}\right)$ spectrum of $(S)-9 \quad \mathrm{~S} 17$ 
Figure $\mathrm{S} 14 .{ }^{1} \mathrm{H}$ NMR (400 MHz, $\left.\mathrm{CD}_{3} \mathrm{OD}\right)$ spectrum of $(R)-9 \quad \mathrm{~S} 18$

Figure S15. ${ }^{1} \mathrm{H}$ NMR (400 MHz, $\left.\mathrm{CD}_{3} \mathrm{OD}\right)$ spectrum of $10 \quad \mathrm{~S} 19$

Figure $\mathrm{S} 16 .{ }^{1} \mathrm{H} \mathrm{NMR}\left(400 \mathrm{MHz}, \mathrm{CD}_{3} \mathrm{OD}\right)$ spectrum of $11 \quad \mathrm{~S} 20$

Figure $\mathrm{S} 17 .{ }^{1} \mathrm{H} \mathrm{NMR}\left(400 \mathrm{MHz}, \mathrm{CD}_{3} \mathrm{OD}\right)$ spectrum of synthetic kinenzoline (1) $\quad \mathrm{S} 21$

Figure $\mathbf{S} 18 .{ }^{13} \mathrm{C}\left\{{ }^{1} \mathrm{H}\right\} \mathrm{NMR}\left(100 \mathrm{MHz}, \mathrm{CDCl}_{3}\right)$ spectrum of $2 \quad \mathrm{~S} 22$

Figure $\mathrm{S} 19 .{ }^{13} \mathrm{C}\left\{{ }^{1} \mathrm{H}\right\} \mathrm{NMR}\left(100 \mathrm{MHz}, \mathrm{CD}_{3} \mathrm{OD}\right)$ spectrum of $4 \quad$ S23

Figure $\mathbf{S} 20 .{ }^{13} \mathrm{C}\left\{{ }^{1} \mathrm{H}\right\} \mathrm{NMR}\left(100 \mathrm{MHz}, \mathrm{CD}_{3} \mathrm{OD}\right)$ spectrum of 5

Figure $\mathrm{S} 21 .{ }^{13} \mathrm{C}\{1 \mathrm{H}\} \mathrm{NMR}\left(100 \mathrm{MHz}, \mathrm{CDCl}_{3}\right)$ spectrum of 7

Figure $\mathbf{S} 22 .{ }^{13} \mathrm{C}\left\{{ }_{1} \mathrm{H}\right\} \mathrm{NMR}\left(100 \mathrm{MHz}, \mathrm{CD}_{3} \mathrm{OD}\right)$ spectrum of $(\boldsymbol{S})-9 \quad \mathrm{~S} 26$

Figure S23. ${ }^{13} \mathrm{C}\{1 \mathrm{H}\} \mathrm{NMR}\left(100 \mathrm{MHz}, \mathrm{CD}_{3} \mathrm{OD}\right)$ spectrum of $(\boldsymbol{R})-\mathbf{9} \quad \mathrm{S} 27$

Figure $\mathrm{S} 24 .{ }^{13} \mathrm{C}\left\{{ }^{1} \mathrm{H}\right\} \mathrm{NMR}\left(100 \mathrm{MHz}, \mathrm{CD}_{3} \mathrm{OD}\right)$ spectrum of $10 \quad \mathrm{~S} 28$

Figure $\mathrm{S} 25 .{ }^{13} \mathrm{C}\left\{{ }^{1} \mathrm{H}\right\} \mathrm{NMR}\left(100 \mathrm{MHz}, \mathrm{CD}_{3} \mathrm{OD}\right)$ spectrum of $11 \quad \mathrm{~S} 29$

Figure S26. ${ }^{13} \mathrm{C}\{1 \mathrm{H}\} \mathrm{NMR}\left(100 \mathrm{MHz}, \mathrm{CD}_{3} \mathrm{OD}\right)$ spectrum of synthetic kinenzoline (1) S30 
Figure $\mathrm{S} 1 .{ }^{1} \mathrm{H}$ NMR (400 MHz, $\left.\mathrm{CD}_{3} \mathrm{OD}\right)$ spectrum of kinenzoline (1)

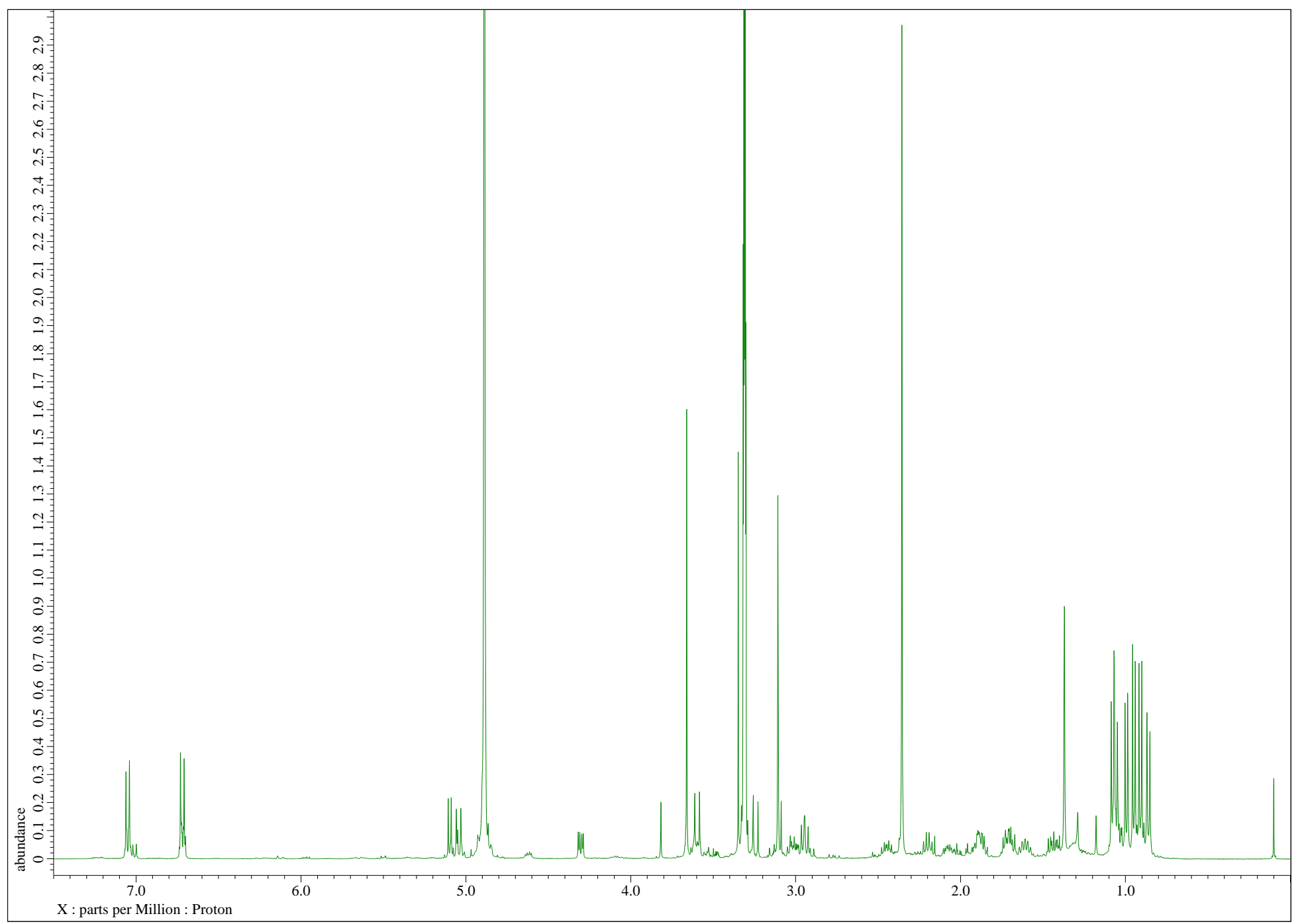


Figure $\mathrm{S} 2 .{ }^{13} \mathrm{C}\left\{{ }^{1} \mathrm{H}\right\} \mathrm{NMR}\left(100 \mathrm{MHz}, \mathrm{CD}_{3} \mathrm{OD}\right)$ spectrum of kinenzoline (1)

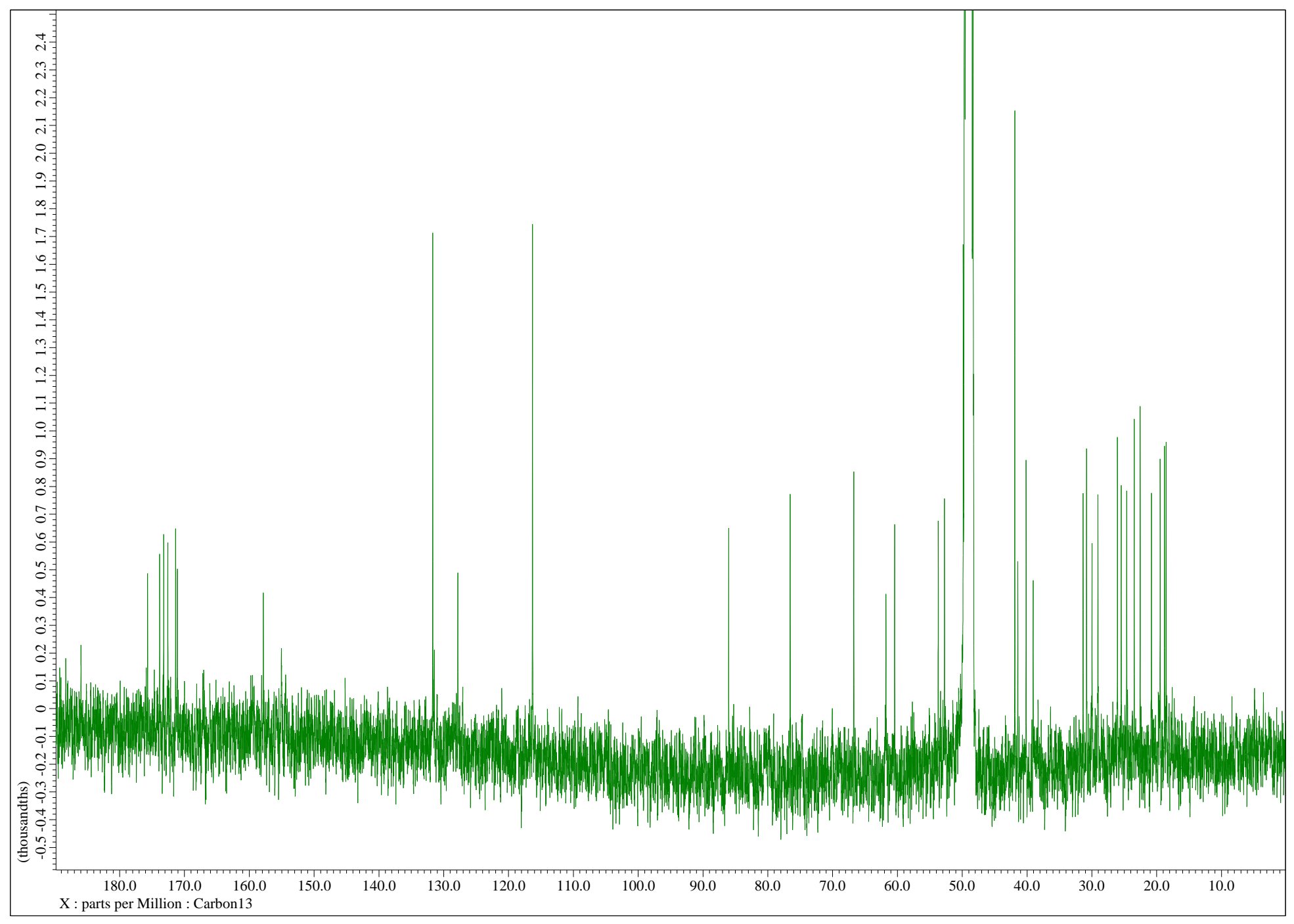


Figure S3. COSY (400 MHz, CD 30 D) spectrum of kinenzoline (1)

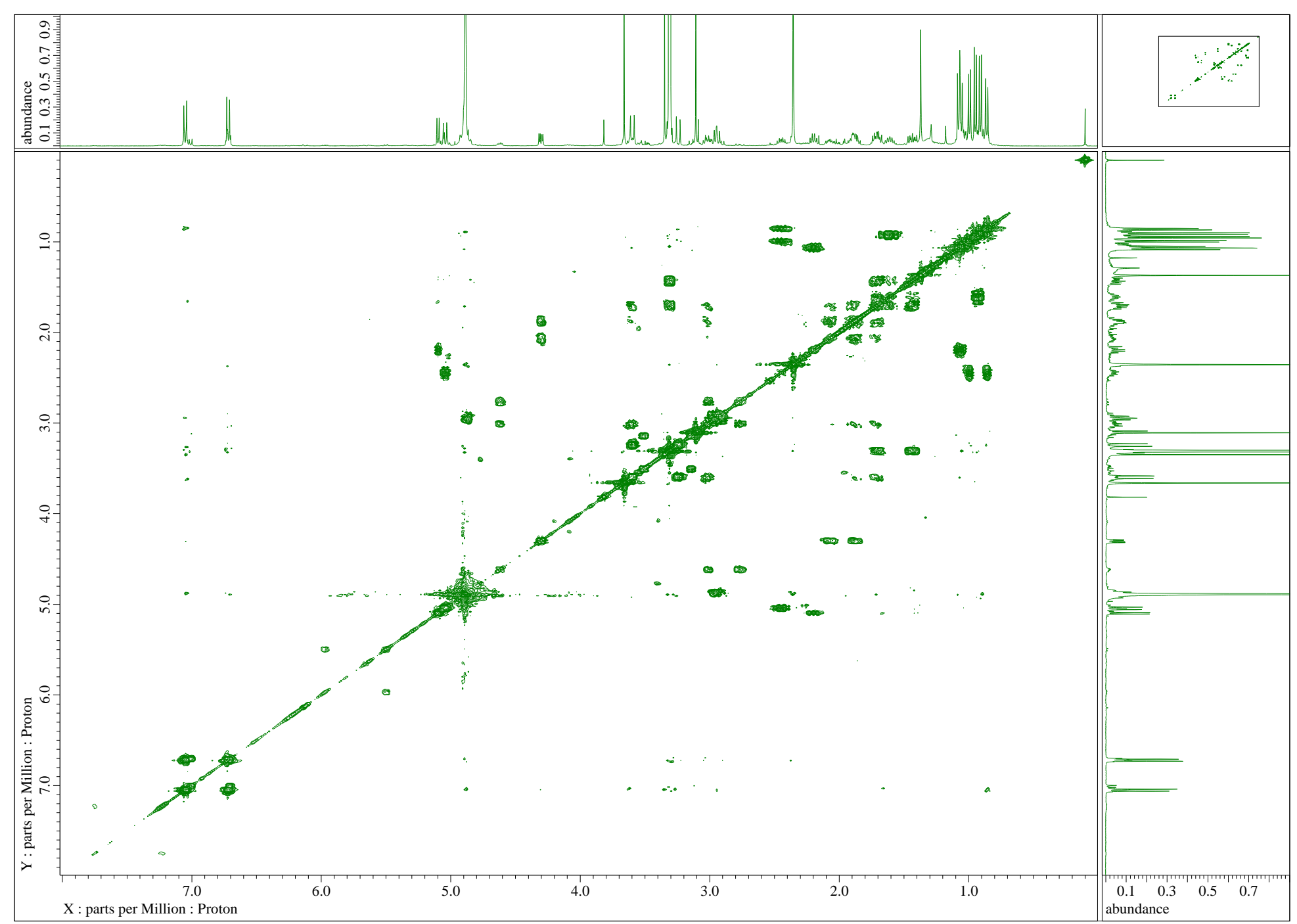


Figure S4. $\mathrm{HMQC}\left(400 \mathrm{MHz}, \mathrm{CD}_{3} \mathrm{OD}\right.$ ) spectrum of kinenzoline (1)

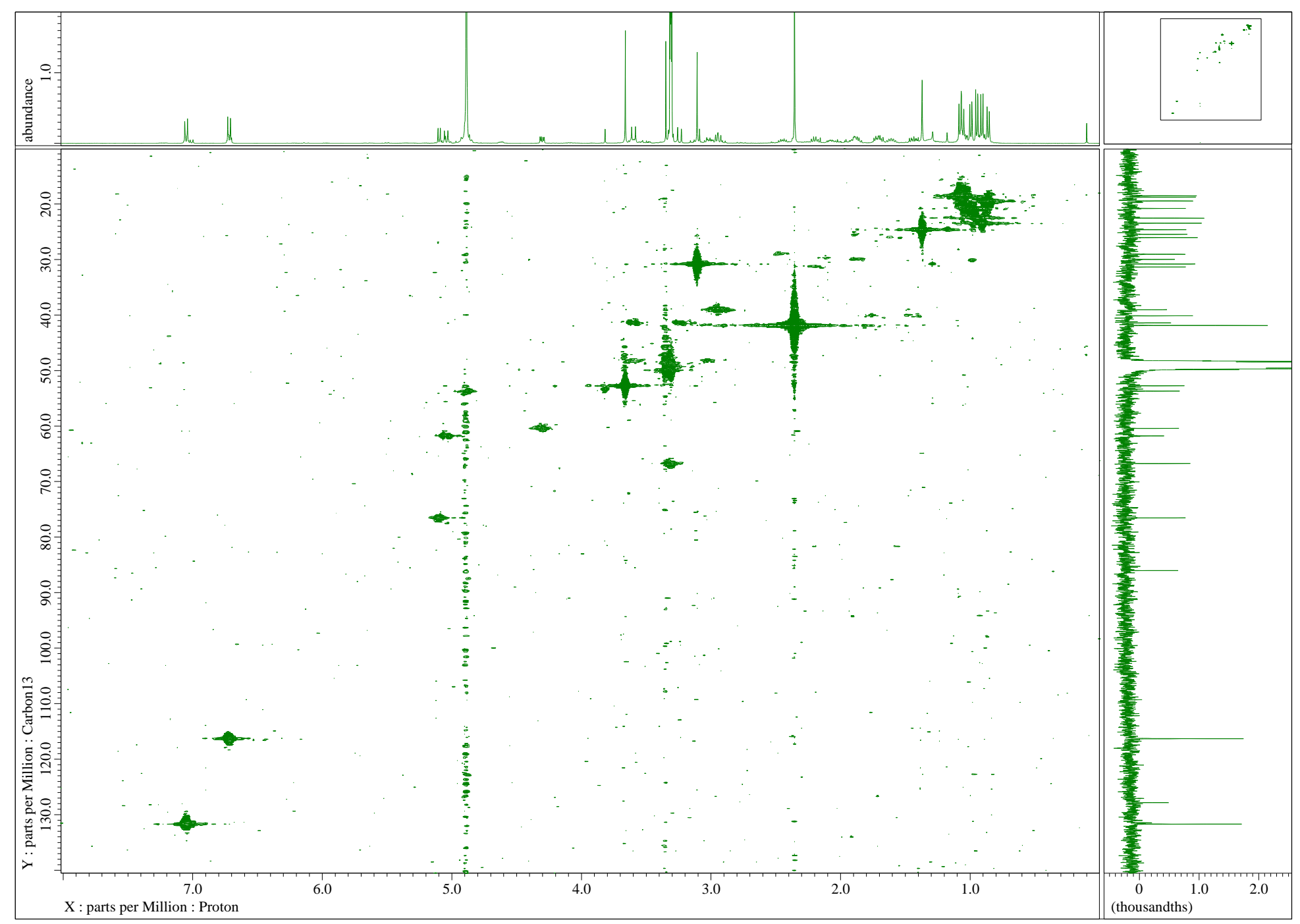


Figure S5. HMBC (400 MHz, CD ${ }_{3} \mathrm{OD}$ ) spectrum of kinenzoline (1)

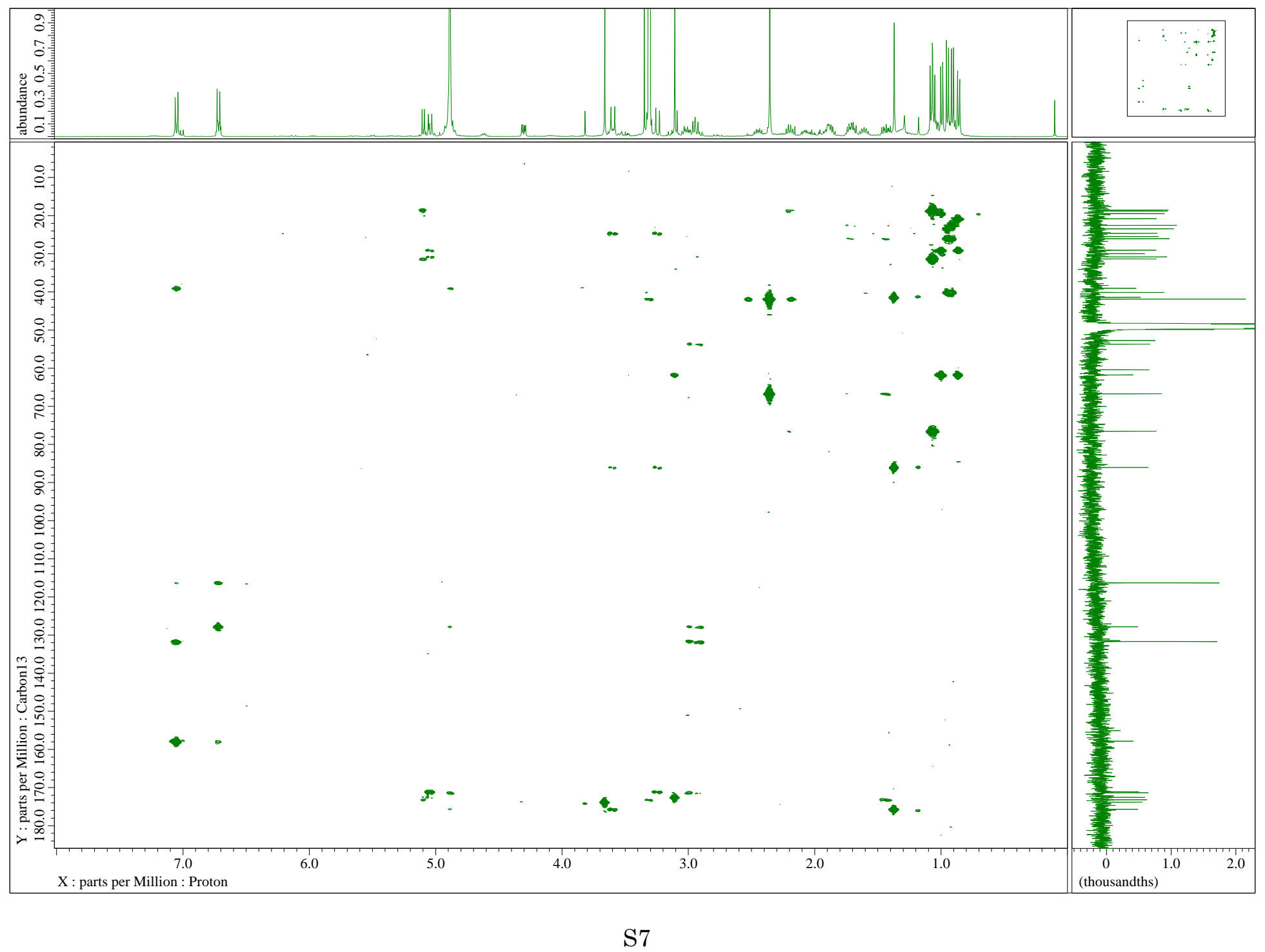


Figure S6. NOESY (400 MHz, $\mathrm{CD}_{3} \mathrm{OD}$ ) spectrum of kinenzoline (1)

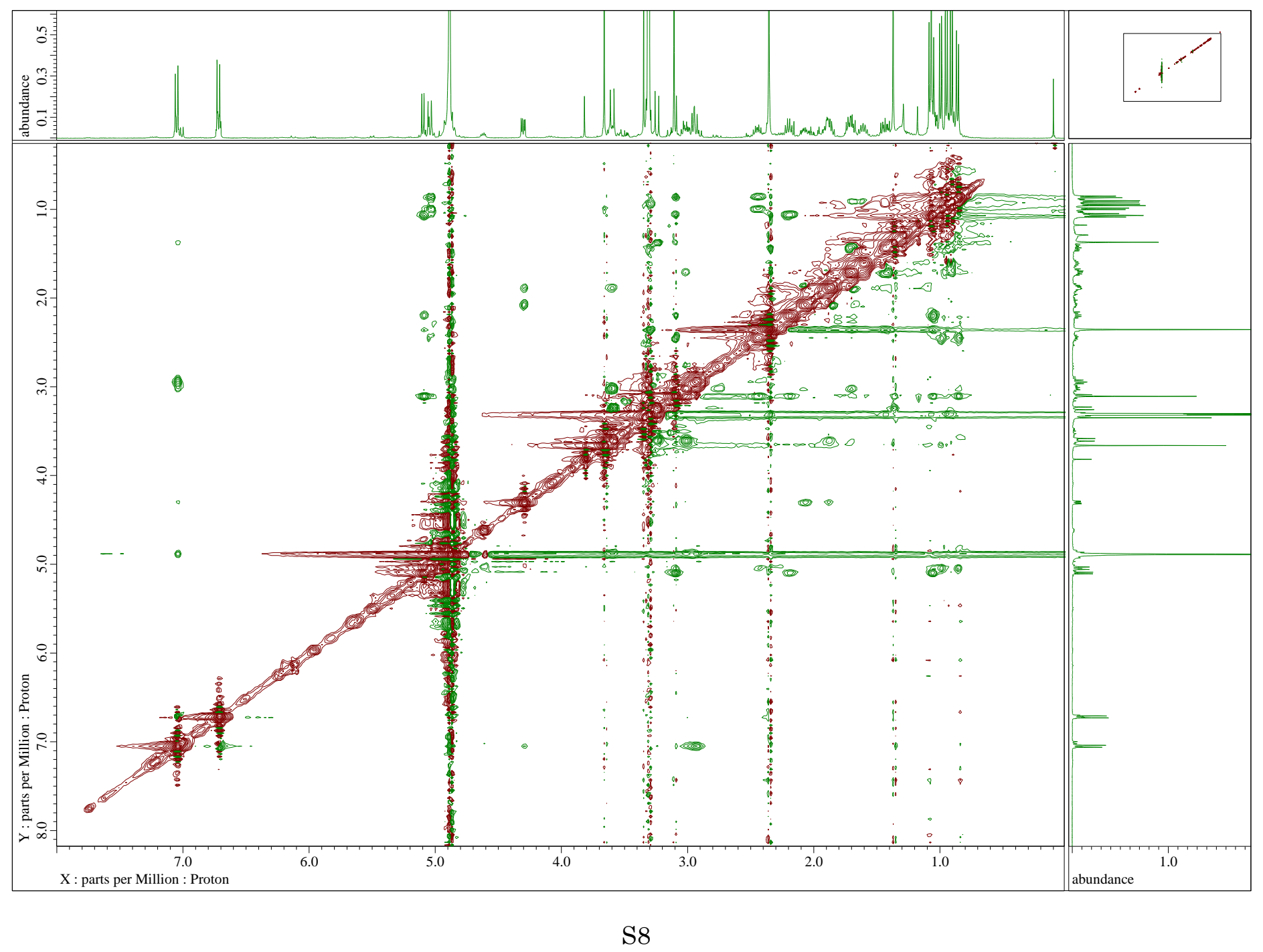


Figure S7. Determination of the absolute configuration of each amino acids in kinenzoline (1)

Pro: column, DAICEL CHIRALPAK $(\mathrm{MA}+)(\phi 4.6 \times 50 \mathrm{~mm})$; flow rate $1.0 \mathrm{~mL} / \mathrm{min}$; detection at $254 \mathrm{~nm}$; solvent, $2.0 \mathrm{mM} \mathrm{CuSO}_{4}$.

tR ( $\min ):$ Authentic samples: D-Pro (2.5), L-Pro (4.3)

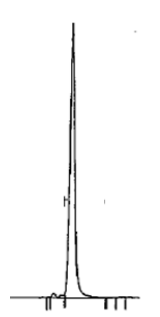

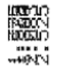

D-Pro

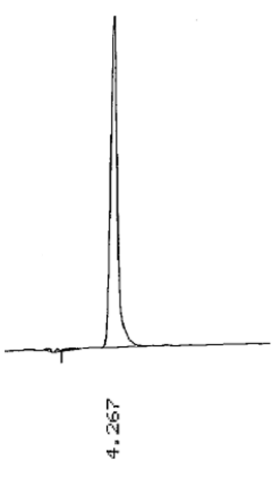

L-Pro

matched

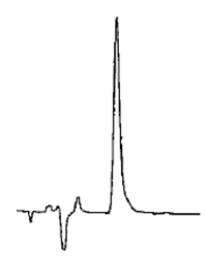

Pro from 1

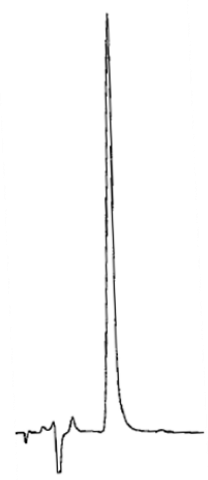

Pro from 1

L-Pro

Tyr: column, DAICEL CHIRALPAK (MA+) $(\phi 4.6 \times 50 \mathrm{~mm})$; flow rate $1.0 \mathrm{~mL} / \mathrm{min}$; detection at $254 \mathrm{~nm}$; solvent, $5 \% \mathrm{MeOH}+2.0 \mathrm{mM} \mathrm{CuSO}_{4}$.

tR (min): Authentic samples: D-Tyr (6.7), L-Tyr (9.6)

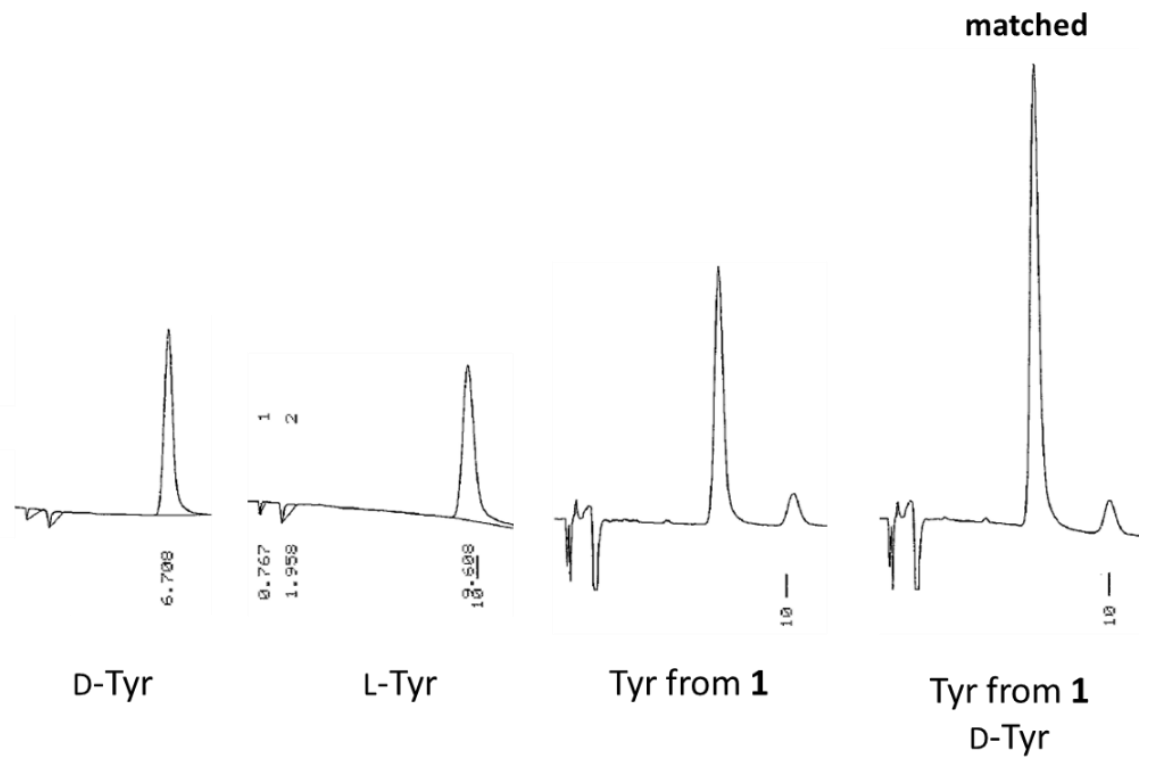


2-methylcysteic acid from 2-MeTzn from 1: column, DAICEL CHIRALPAK (MA+) $(\phi 4.6$ $\times 50 \mathrm{~mm}$ ); flow rate $1.0 \mathrm{~mL} / \mathrm{min}$; detection at $254 \mathrm{~nm}$; solvent, $2.0 \mathrm{mM} \mathrm{CuSO}$.

th (min): Authentic samples: (S)-2-methylcysteic acid (4.9), (R)-2-methylcystiec acid (5.8)

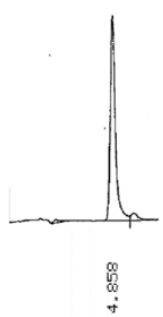

(S)-2-methylcysteic acid

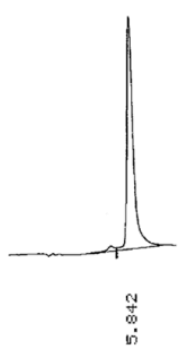

(R)-2-methylcysteic acid

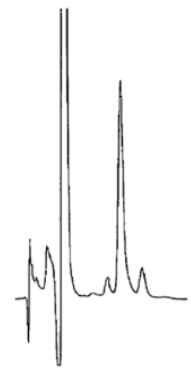

2-methylcysteic acid from 1 2-methylcysteic acid from 1

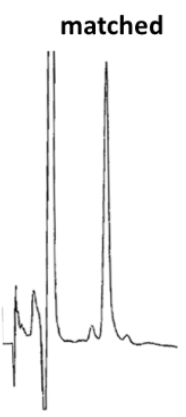

(R)-2-methylcysteic acid

$N$-Me-Val: column, DAICEL CHIRALPAK $(\mathrm{MA}+)(\phi 4.6 \times 50 \mathrm{~mm})$; flow rate $1.0 \mathrm{~mL} / \mathrm{min}$; detection at $254 \mathrm{~nm}$; solvent, $2.0 \mathrm{mM} \mathrm{CuSO}_{4}$.

tR (min): Authentic samples: $N$-Me-D-Val (3.3), $N$-Me-L-Val (5.2)

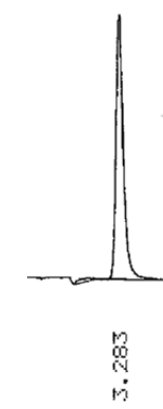

$N-M e-D-V a l$

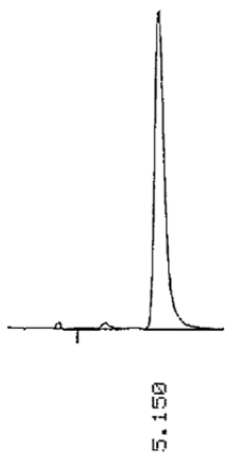

$N$-Me-L-Val

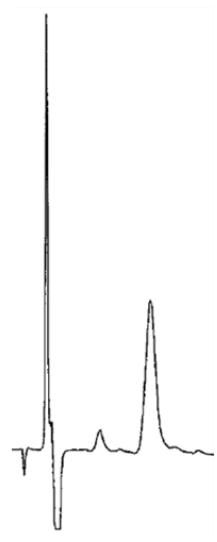

$N$-Me-Val from 1

\section{matched}

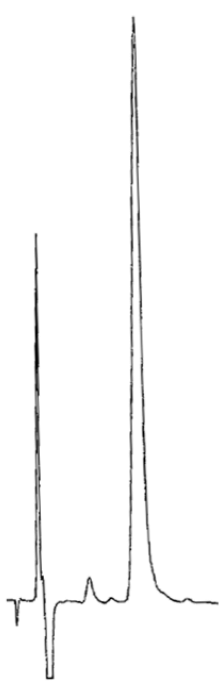

N-Me-Val from 1

$N$-Me-L-Val 
Valic acid: column, DAICEL CHIRALPAK $(\mathrm{MA}+)(\phi 4.6 \times 50 \mathrm{~mm})$; flow rate $1.0 \mathrm{~mL} / \mathrm{min}$; detection at $254 \mathrm{~nm}$; solvent, $15 \% \mathrm{MeCN}+2.0 \mathrm{mM} \mathrm{CuSO}_{4}$.

tR (min): Authentic samples: D-Valic acid (5.4), L-Valic acid (9.2)

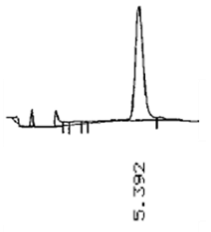

D-Valic acid

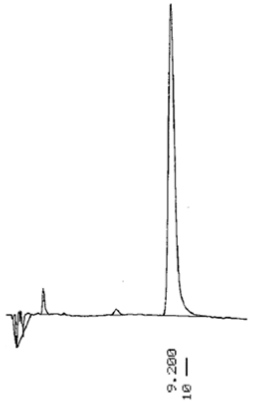

L-Valic acid

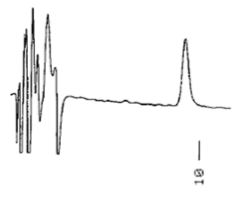

Valic acid from 1

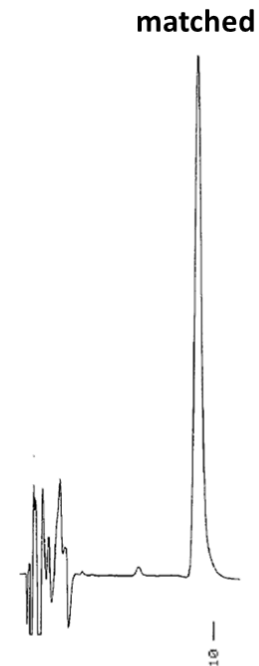

Valic acid from 1 L-Valic acid

$N, N$-diMe-Leu: column, DAICEL CHIRALPAK $(\mathrm{MA}+)(\phi 4.6 \times 50 \mathrm{~mm})$; flow rate 1.0 $\mathrm{mL} / \mathrm{min}$; detection at $254 \mathrm{~nm}$; solvent, $10 \% \mathrm{MeOH}+2.0 \mathrm{mM} \mathrm{CuSO}_{4}$.

tR (min): Authentic samples: $N, N$-diMe-D-Leu (6.4), $N, N$-diMe-L-Leu (10.6)

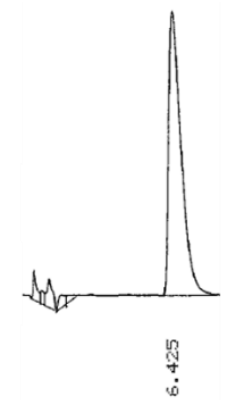

$N, N$-diMe-D-Leu

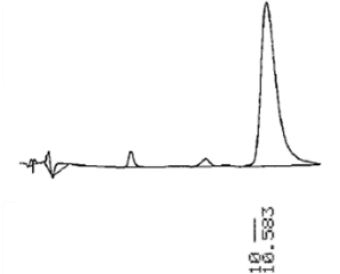

$N, N$-diMe-L-Leu

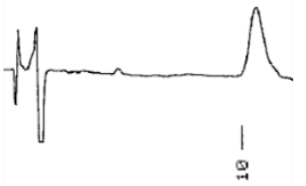

$\mathrm{N}, \mathrm{N}$-diMe-Leu from $1 \mathrm{~N}, \mathrm{~N}$-diMe-Leu from 1 $N, N$-diMe-L-Leu 
Figure S8. Phylogenic tree of the cyanobacterium.

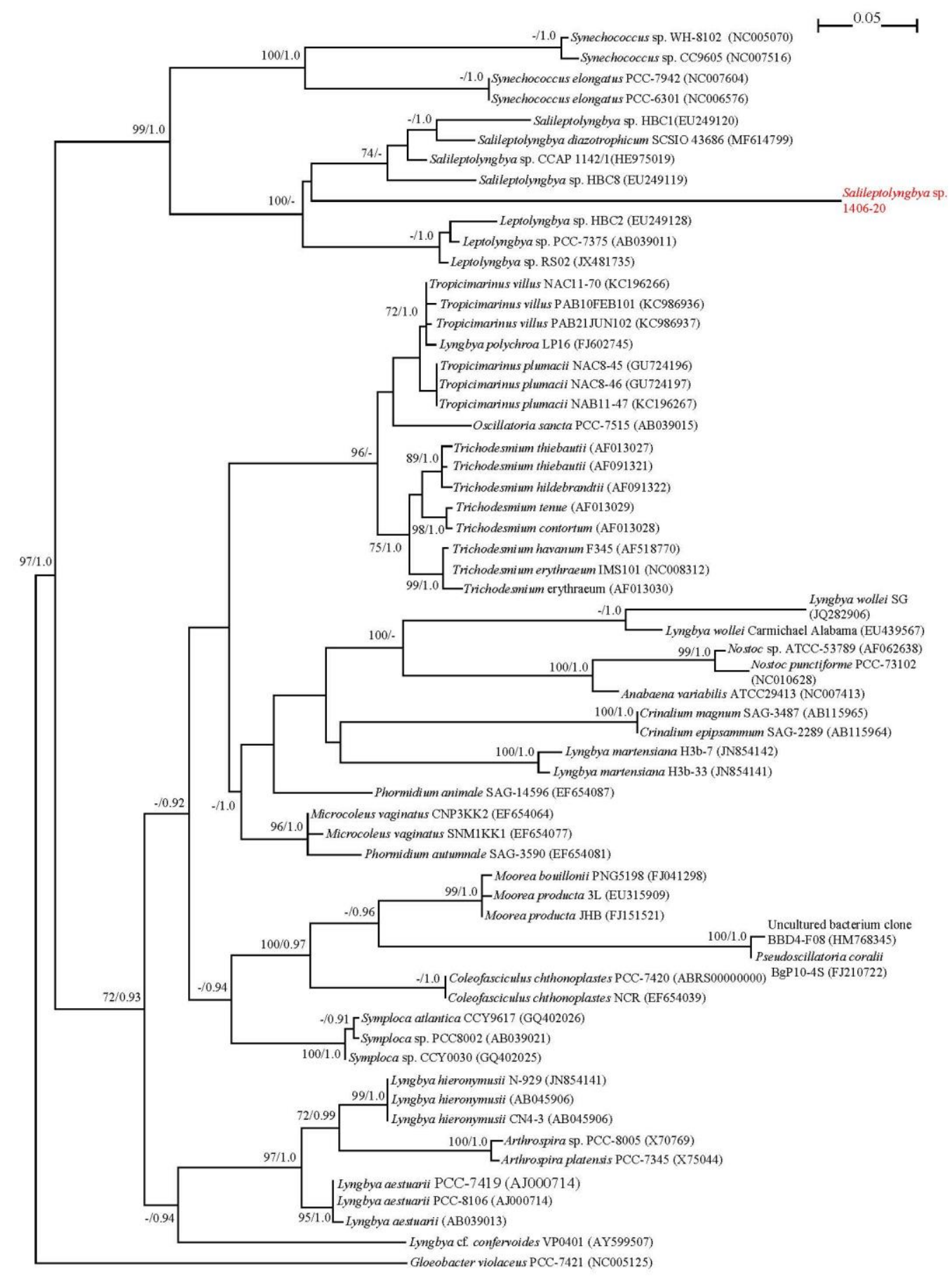

Phylogenetic tree inferred from 16S rDNA sequences using the ML method. The phylogeny is rooted with Gloeobacter violaceus PCC 7421. The numbers at the nodes indicate bootstrap values (values $<70 \%$ are not shown) and posterior probability (values $<0.9$ are not shown) for the ML/Bayesian inference. 
Figure S9. $\left.{ }^{1} \mathrm{H} \mathrm{NMR} \mathrm{(400} \mathrm{MHz,} \mathrm{CDCl}_{3}\right)$ spectrum of 2

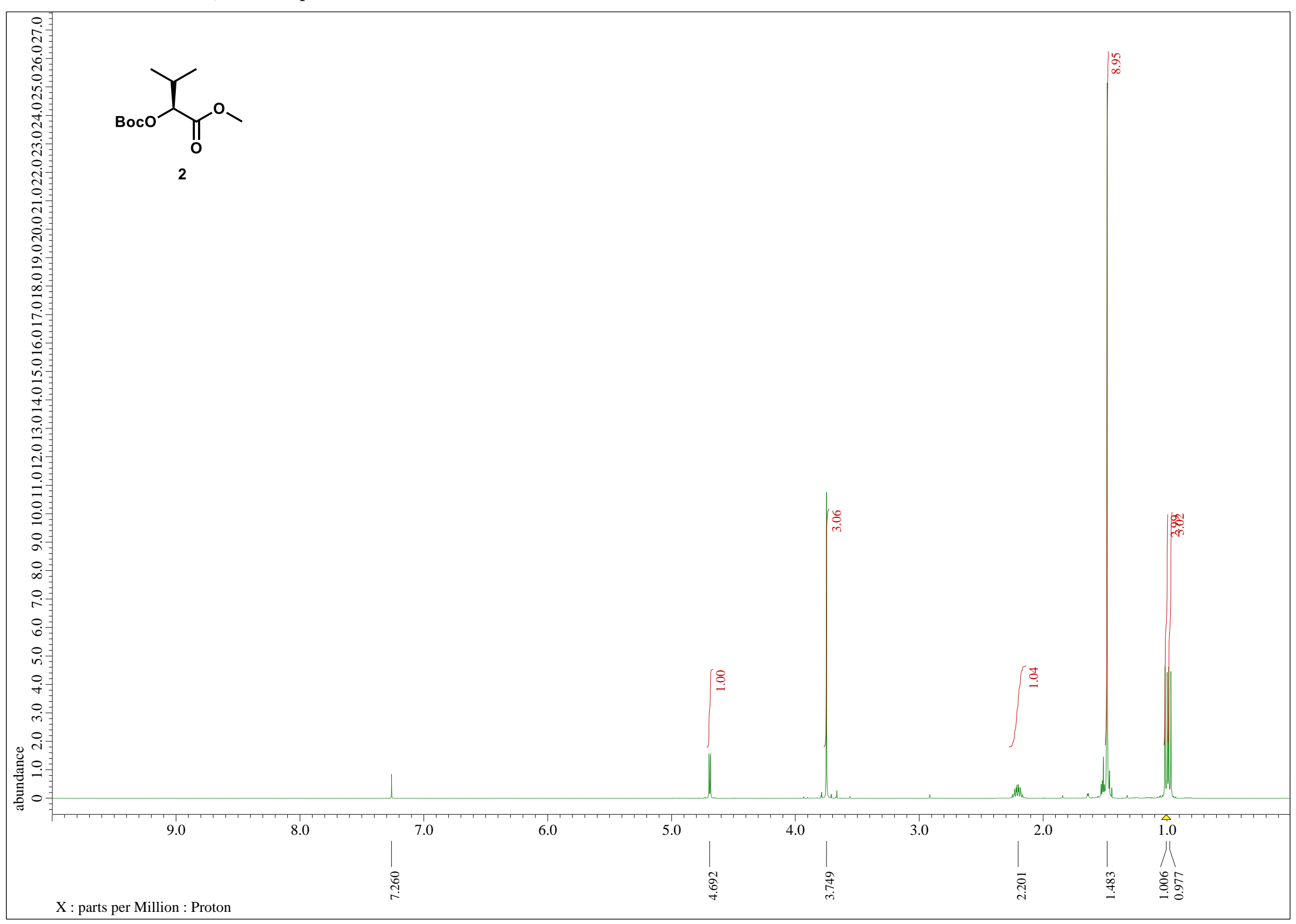


Figure S10. ${ }^{1} \mathrm{H}$ NMR $\left(400 \mathrm{MHz}, \mathrm{CD}_{3} \mathrm{OD}\right)$ spectrum of 4

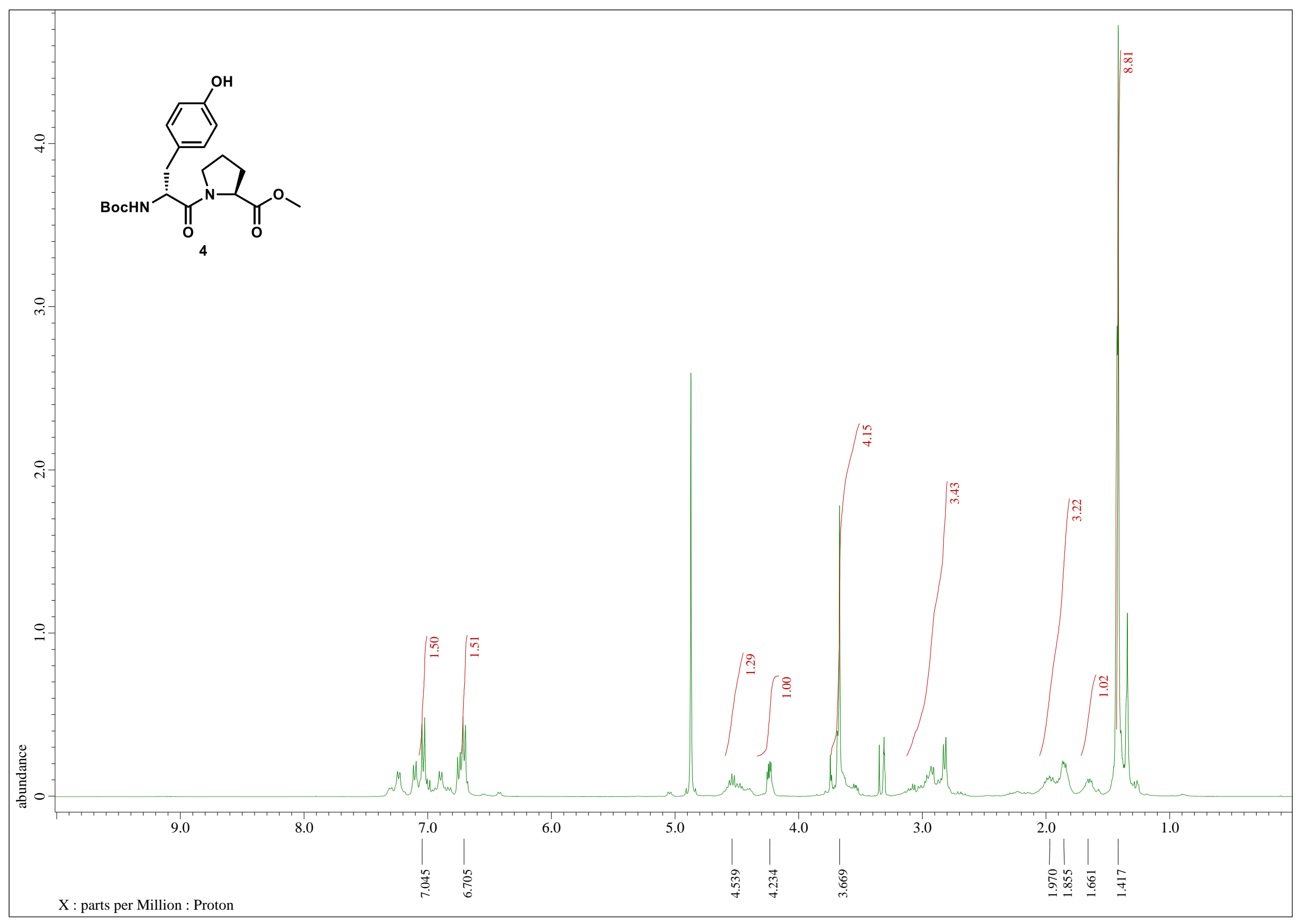


Figure S11. ${ }^{1} \mathrm{H}$ NMR (400 MHz, $\left.\mathrm{CD}_{3} \mathrm{OD}\right)$ spectrum of $\mathbf{5}$

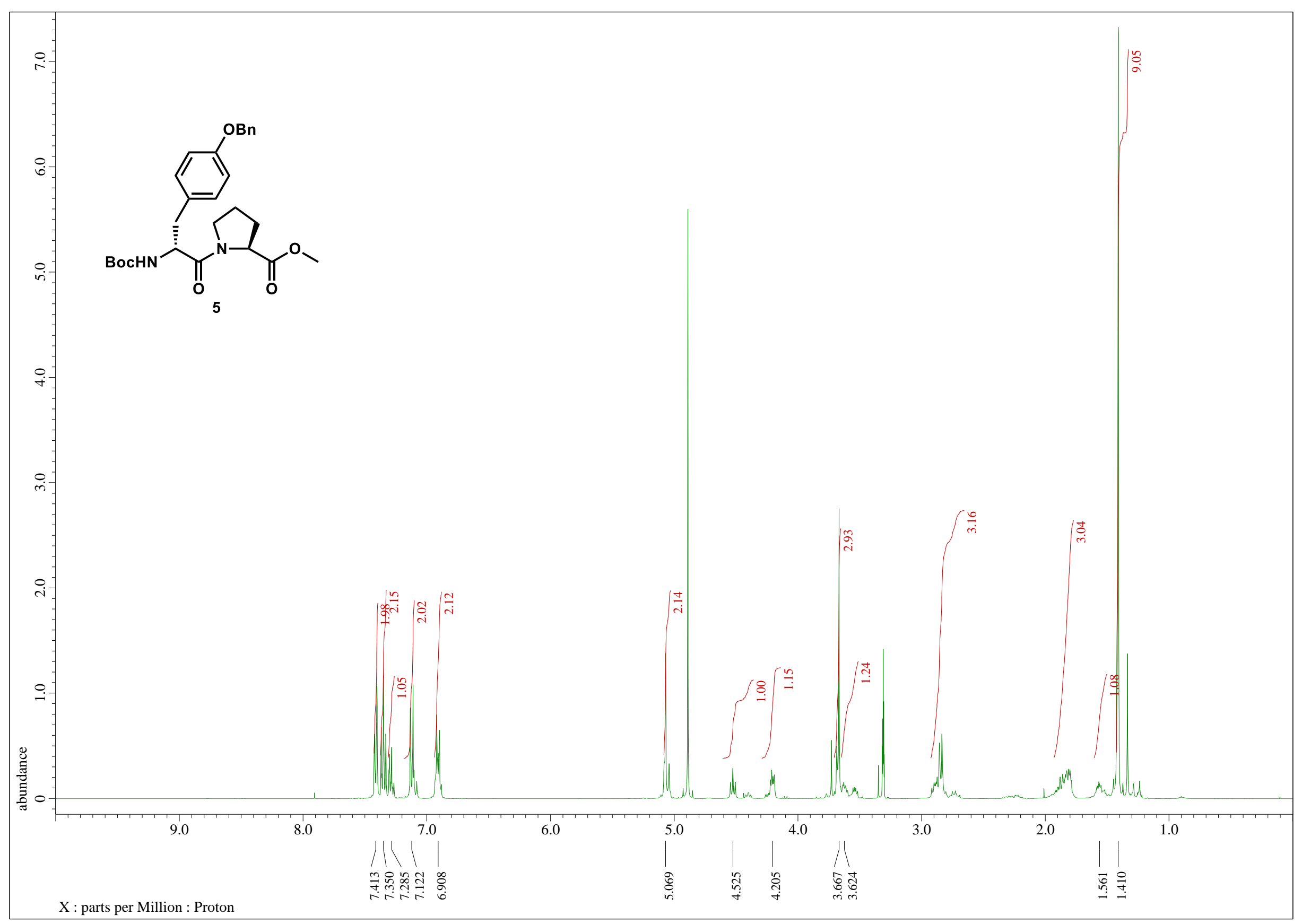


Figure $\mathrm{S} 12 .{ }^{1} \mathrm{H} \mathrm{NMR}\left(400 \mathrm{MHz}, \mathrm{CDCl}_{3}\right)$ spectrum of 7

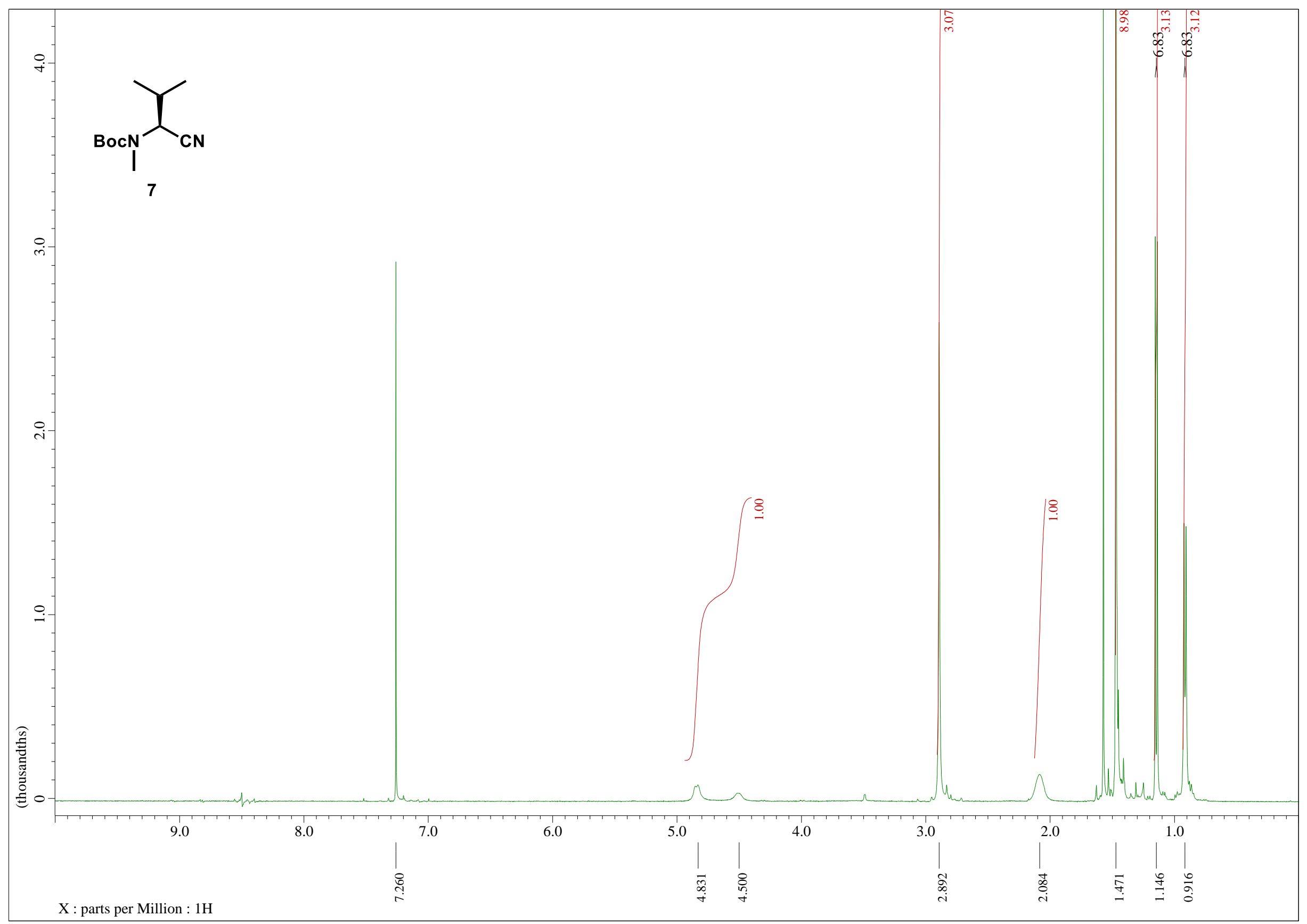


Figure S13. ${ }^{1} \mathrm{H}$ NMR (400 MHz, $\left.\mathrm{CD}_{3} \mathrm{OD}\right)$ spectrum of $(S)-9$

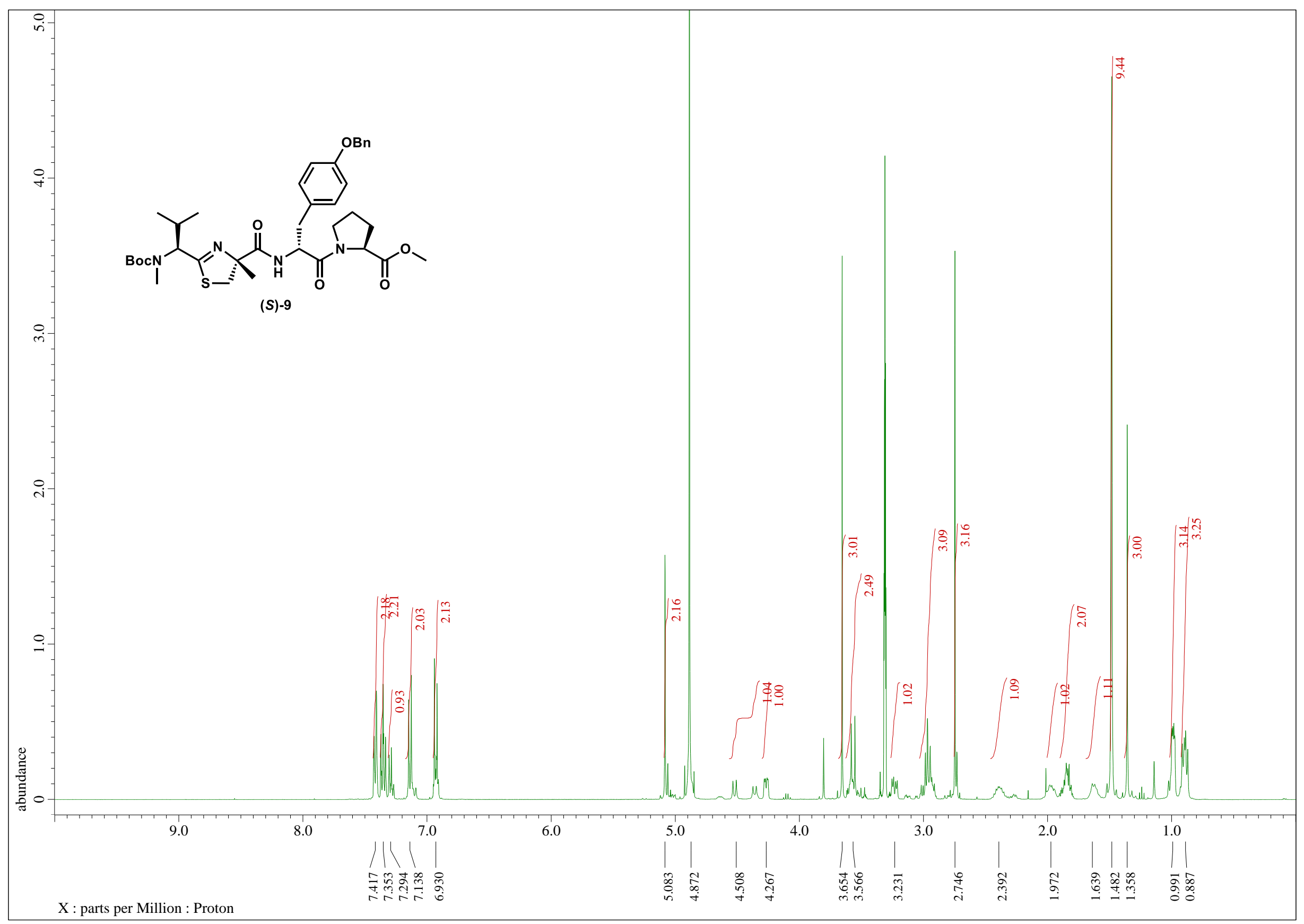


Figure S14. ${ }^{1} \mathrm{H}$ NMR (400 MHz, $\left.\mathrm{CD}_{3} \mathrm{OD}\right)$ spectrum of $(R)$-9

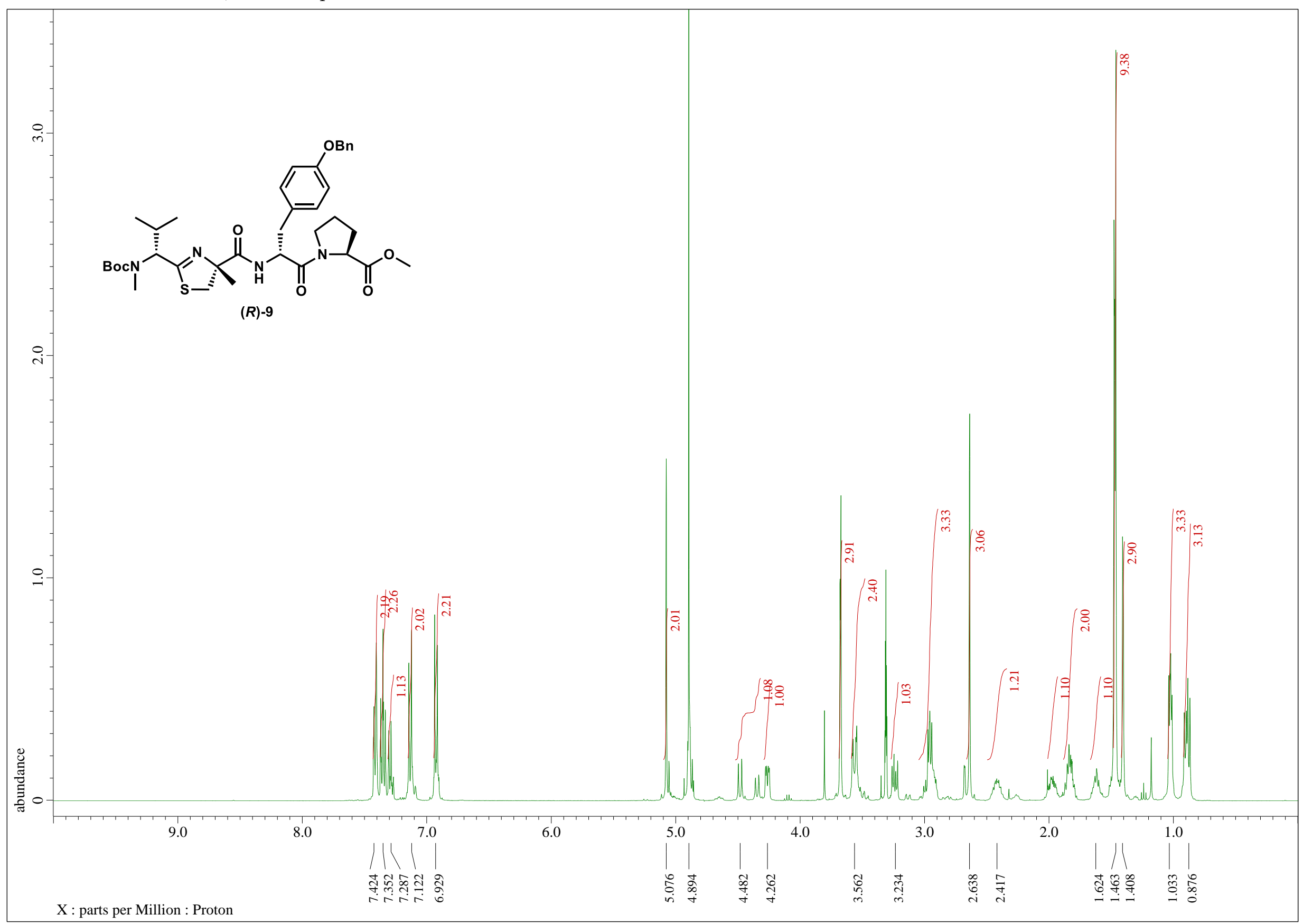


Figure S15. ${ }^{1} \mathrm{H}$ NMR $\left(400 \mathrm{MHz}, \mathrm{CD}_{3} \mathrm{OD}\right)$ spectrum of 10

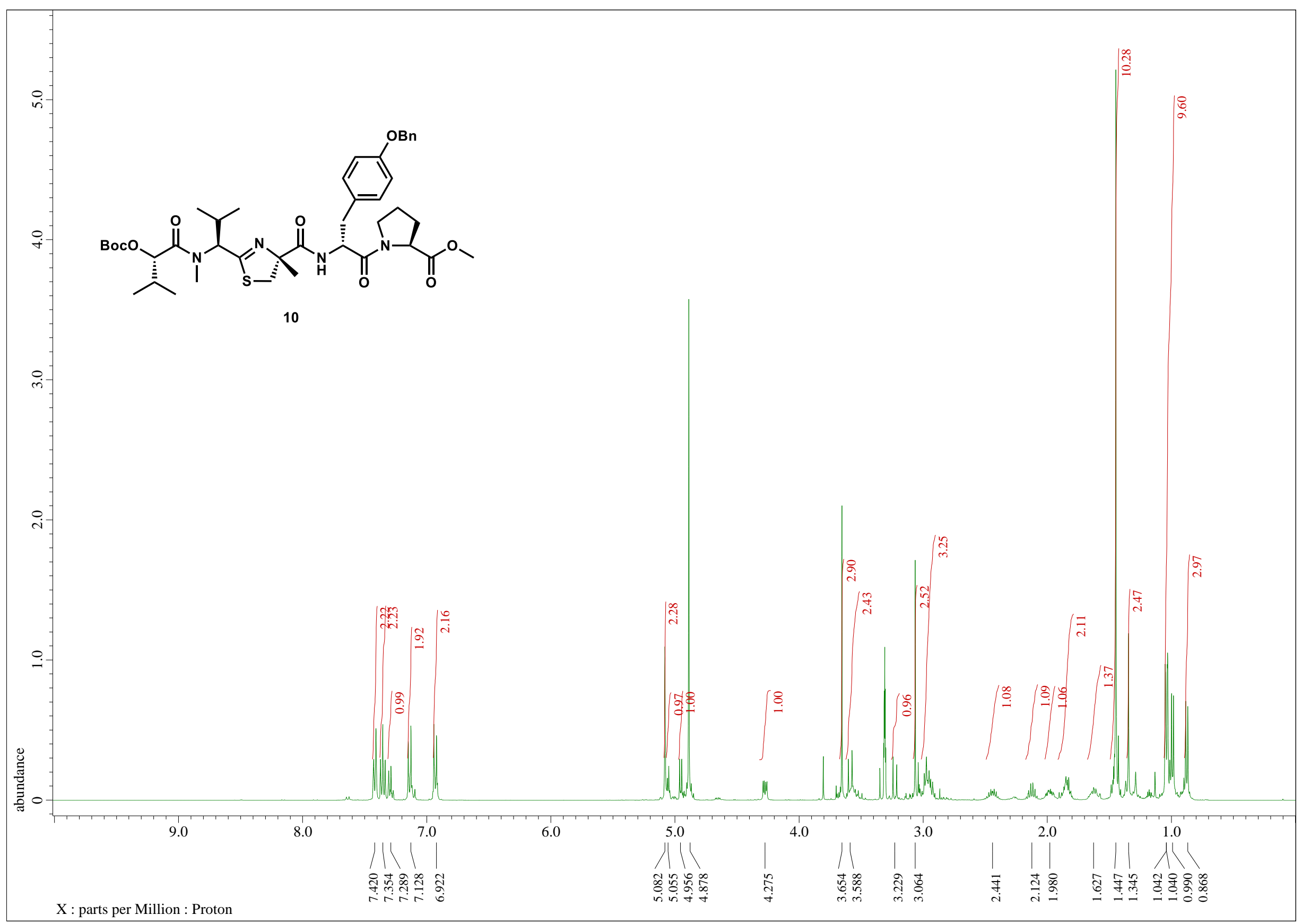


Figure $\mathrm{S} 16 .{ }^{1} \mathrm{H}$ NMR (400 MHz, $\left.\mathrm{CD}_{3} \mathrm{OD}\right)$ spectrum of 11

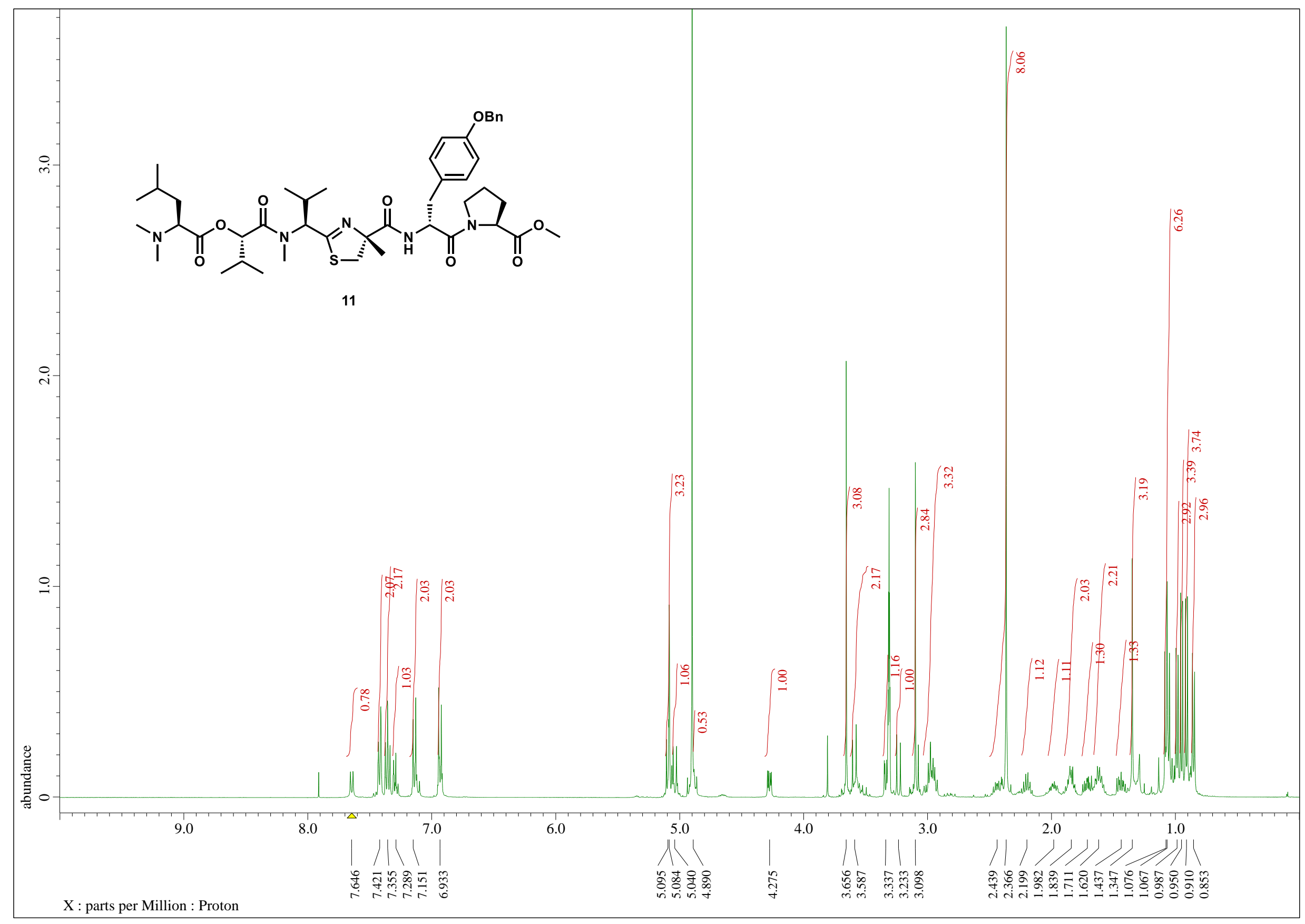


Figure S17. ${ }^{1} \mathrm{H}$ NMR (400 MHz, $\left.\mathrm{CD}_{3} \mathrm{OD}\right)$ spectrum of synthetic kinenzoline (1)

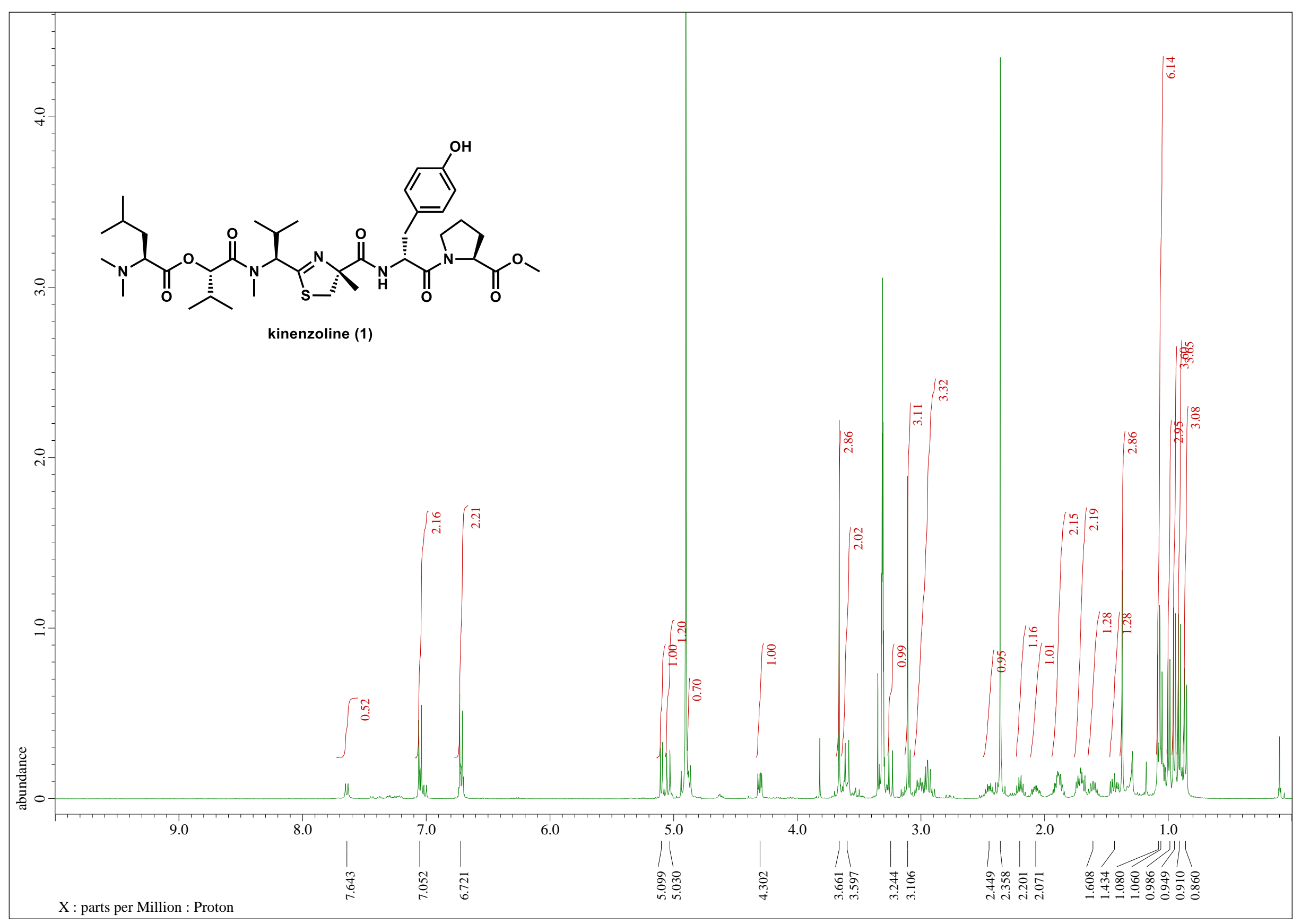


Figure $\mathrm{S} 18 .{ }^{13} \mathrm{C}\{1 \mathrm{H}\} \mathrm{NMR}\left(100 \mathrm{MHz}, \mathrm{CDCl}_{3}\right)$ spectrum of $\mathbf{2}$

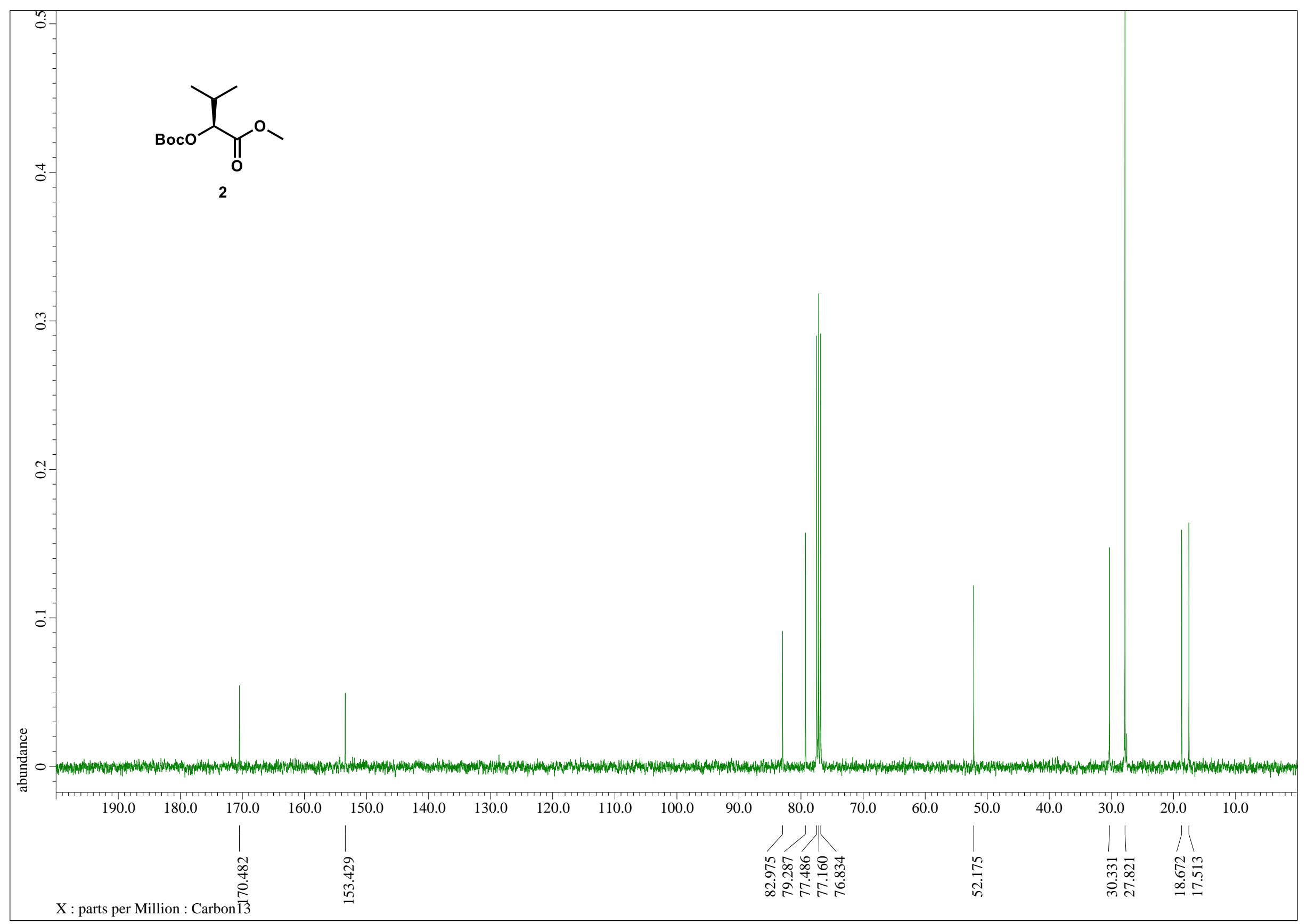


Figure $\mathrm{S} 19 .{ }^{13} \mathrm{C}\left\{{ }^{1} \mathrm{H}\right\} \mathrm{NMR}\left(100 \mathrm{MHz}, \mathrm{CD}_{3} \mathrm{OD}\right)$ spectrum of 4

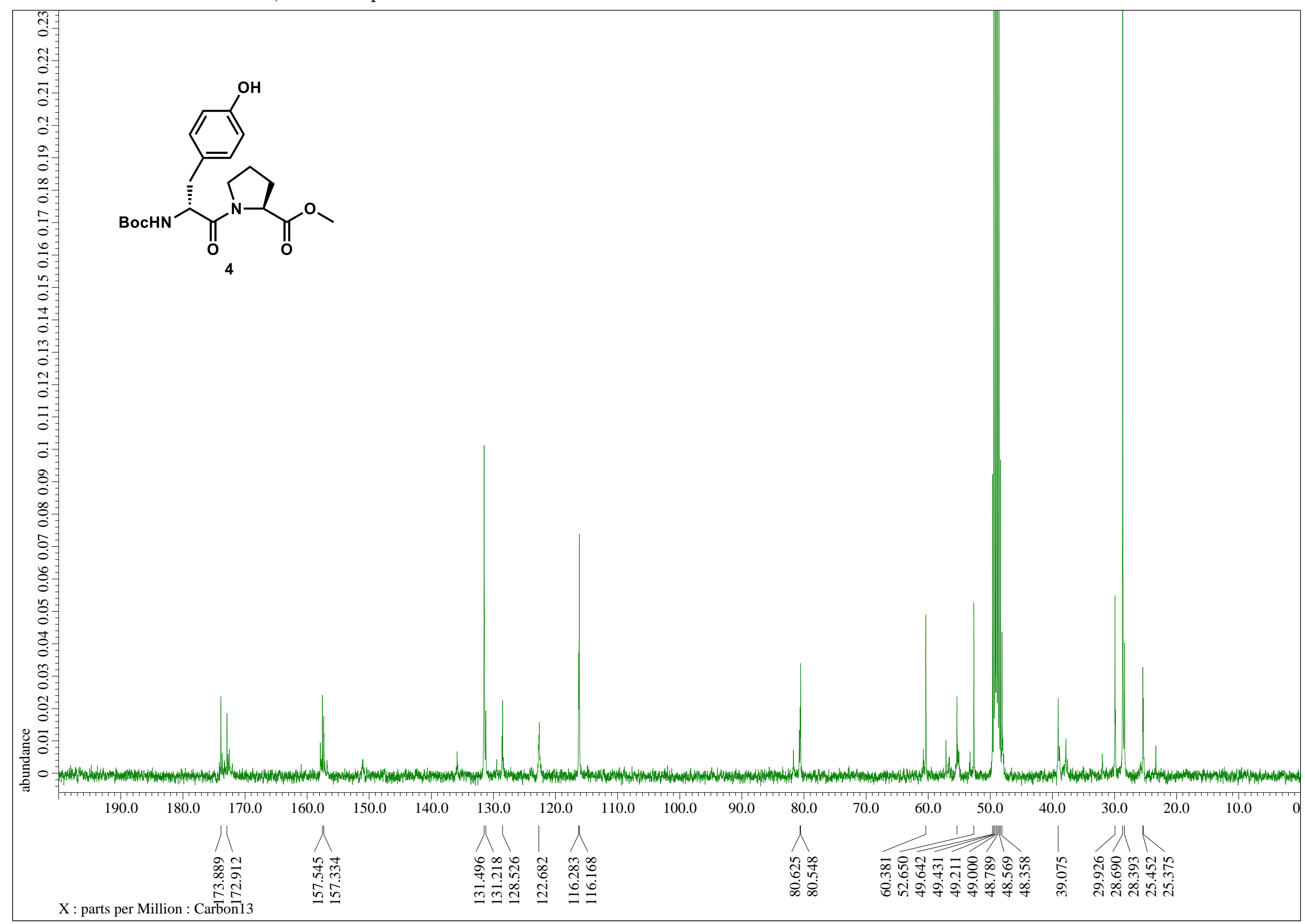


Figure $\mathrm{S} 20 .{ }^{13} \mathrm{C}\{1 \mathrm{H}\} \mathrm{NMR}\left(100 \mathrm{MHz}, \mathrm{CD}_{3} \mathrm{OD}\right)$ spectrum of $\mathbf{5}$

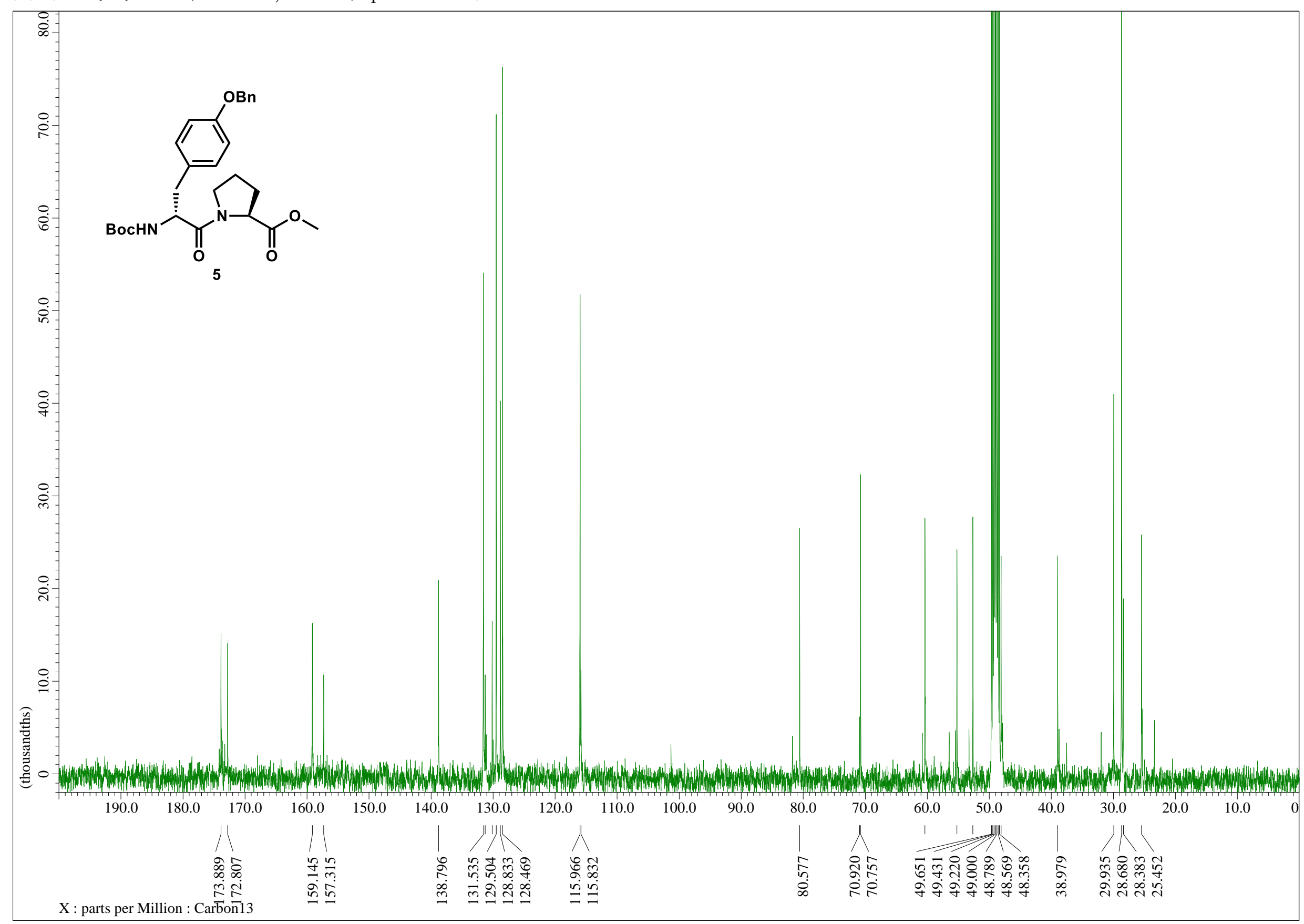


Figure $\mathrm{S} 21 .{ }^{13} \mathrm{C}\{1 \mathrm{H}\} \mathrm{NMR}\left(100 \mathrm{MHz}, \mathrm{CDCl}_{3}\right)$ spectrum of $\mathbf{7}$

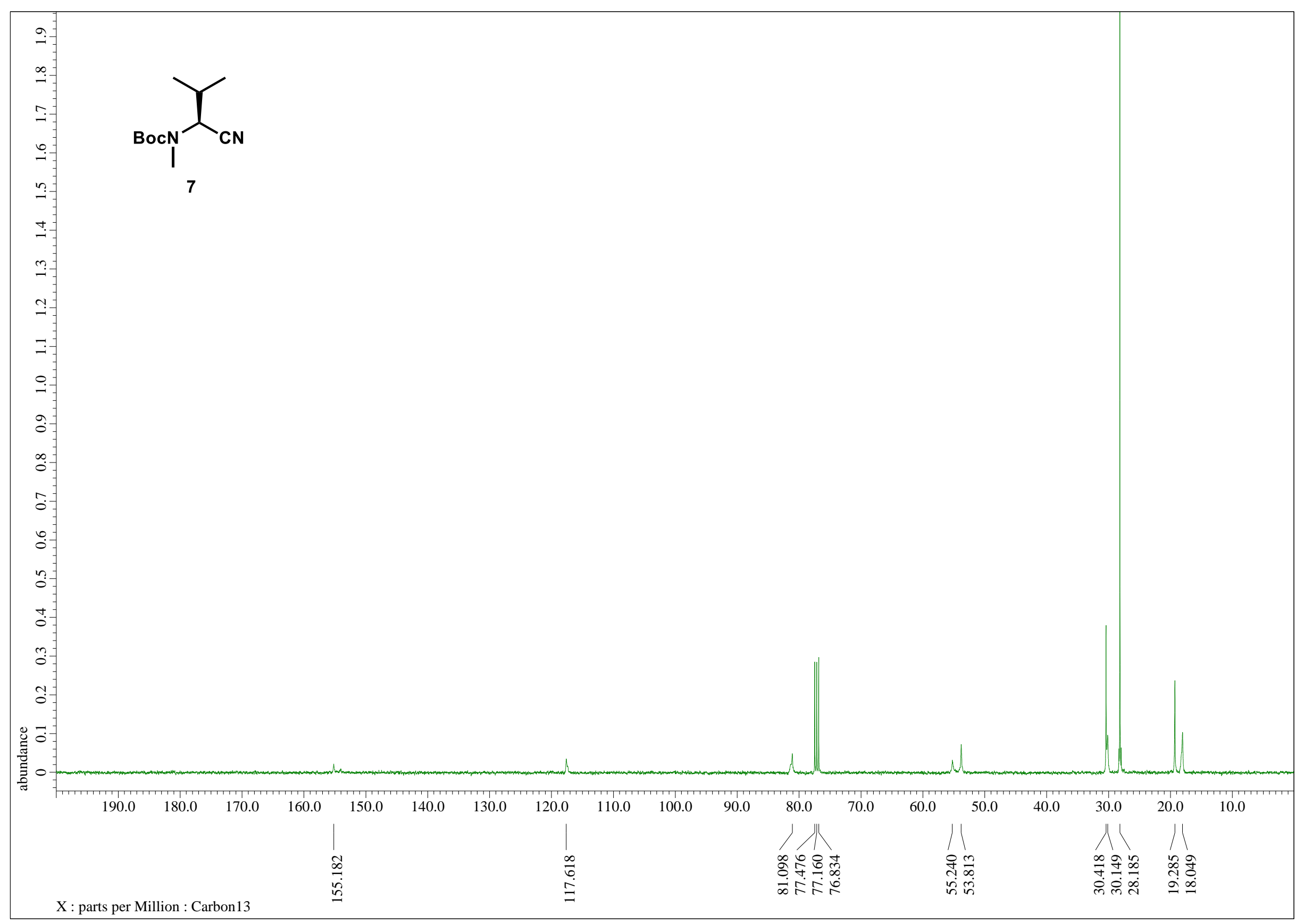


Figure $\mathrm{S} 22 .{ }^{13} \mathrm{C}\{1 \mathrm{H}\} \mathrm{NMR}\left(100 \mathrm{MHz}, \mathrm{CD}_{3} \mathrm{OD}\right)$ spectrum of $(\boldsymbol{S})-\mathbf{9}$

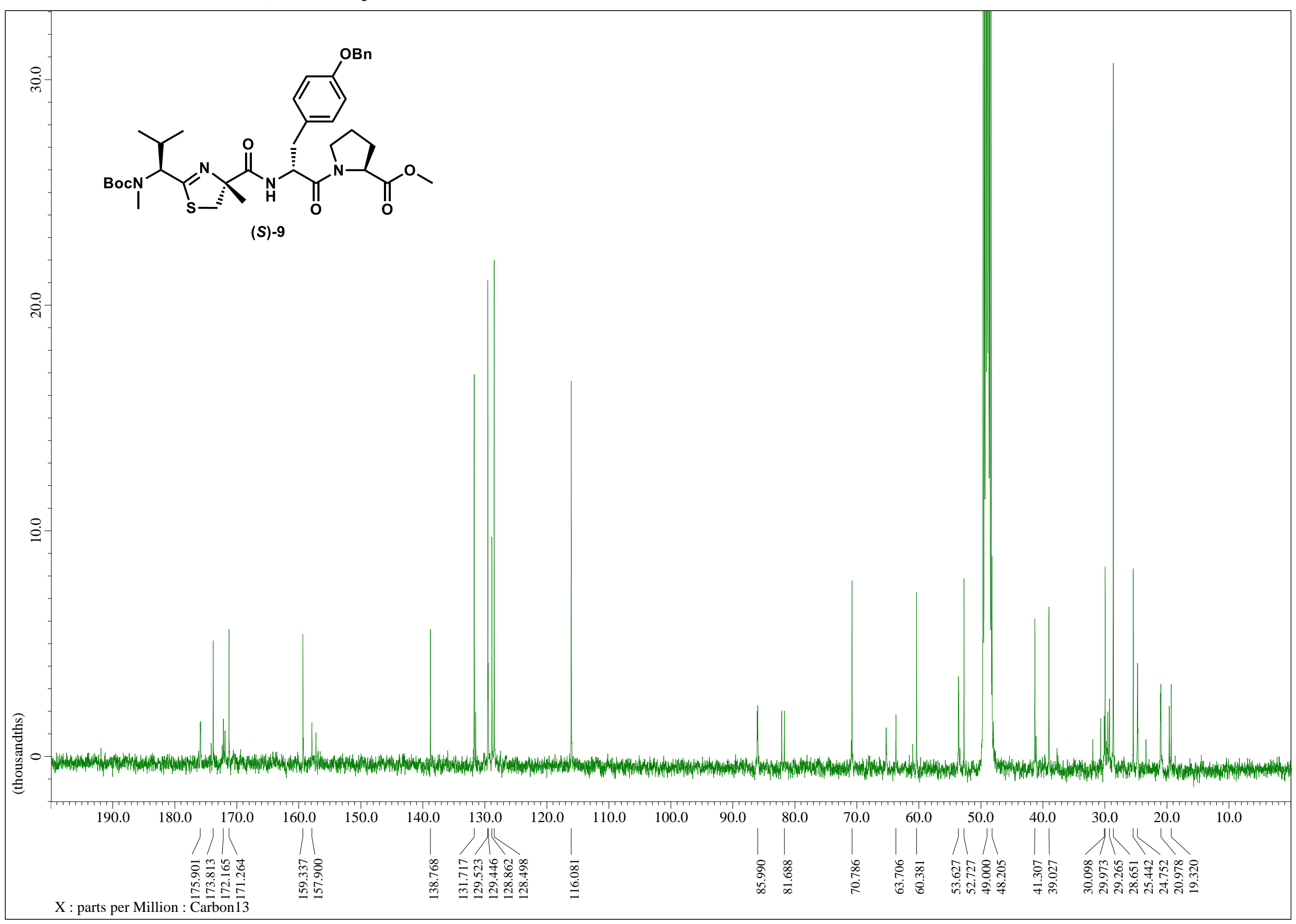


Figure $\mathbf{S} 23 .{ }^{13} \mathrm{C}\left\{{ }^{1} \mathrm{H}\right\} \mathrm{NMR}\left(100 \mathrm{MHz}, \mathrm{CD}_{3} \mathrm{OD}\right)$ spectrum of $(\boldsymbol{R})-\mathbf{9}$

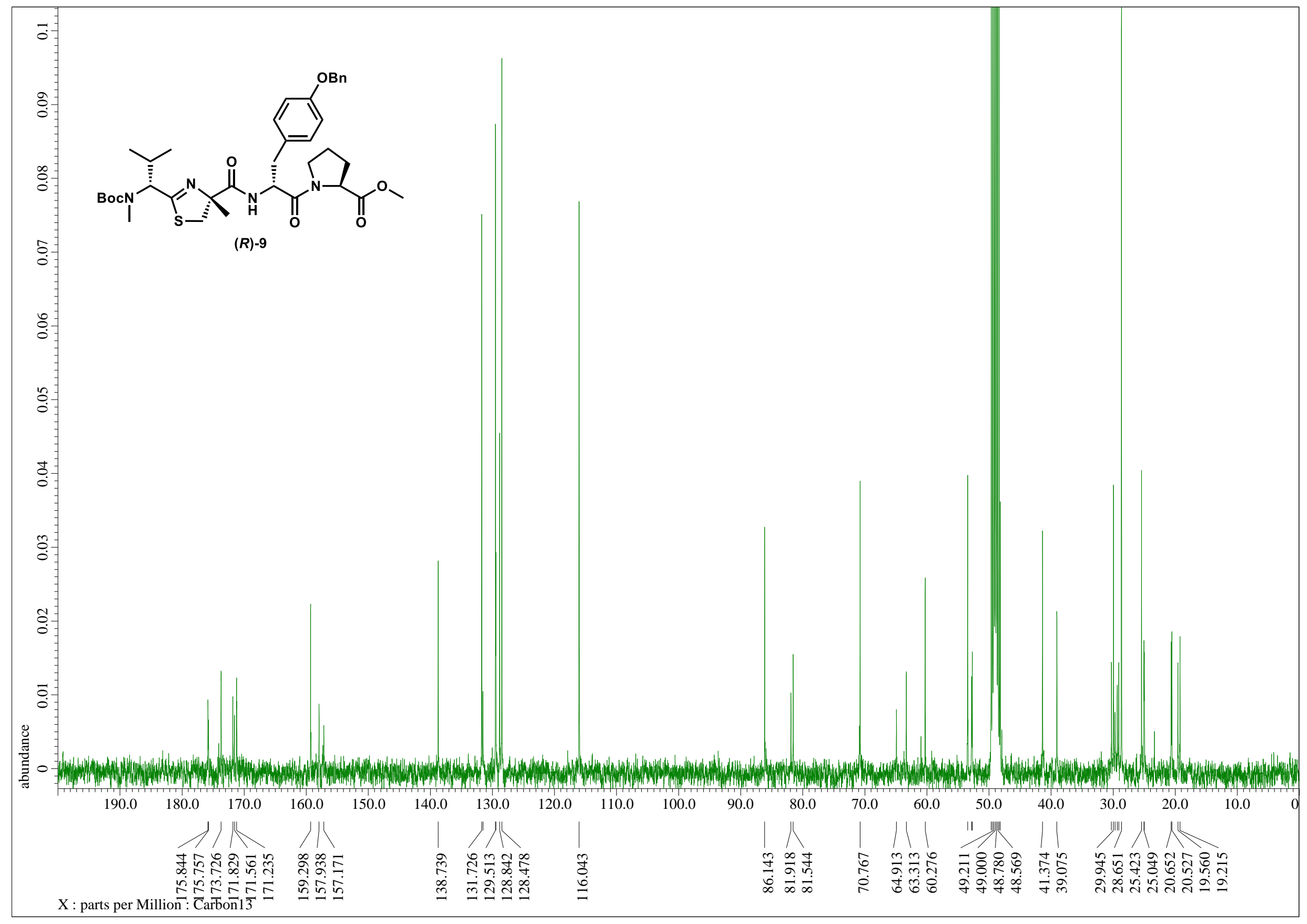


Figure $\mathrm{S}_{24}{ }^{13 \mathrm{C}}\left\{{ }^{1} \mathrm{H}\right\} \mathrm{NMR}\left(100 \mathrm{MHz}, \mathrm{CD}_{3} \mathrm{OD}\right)$ spectrum of 10

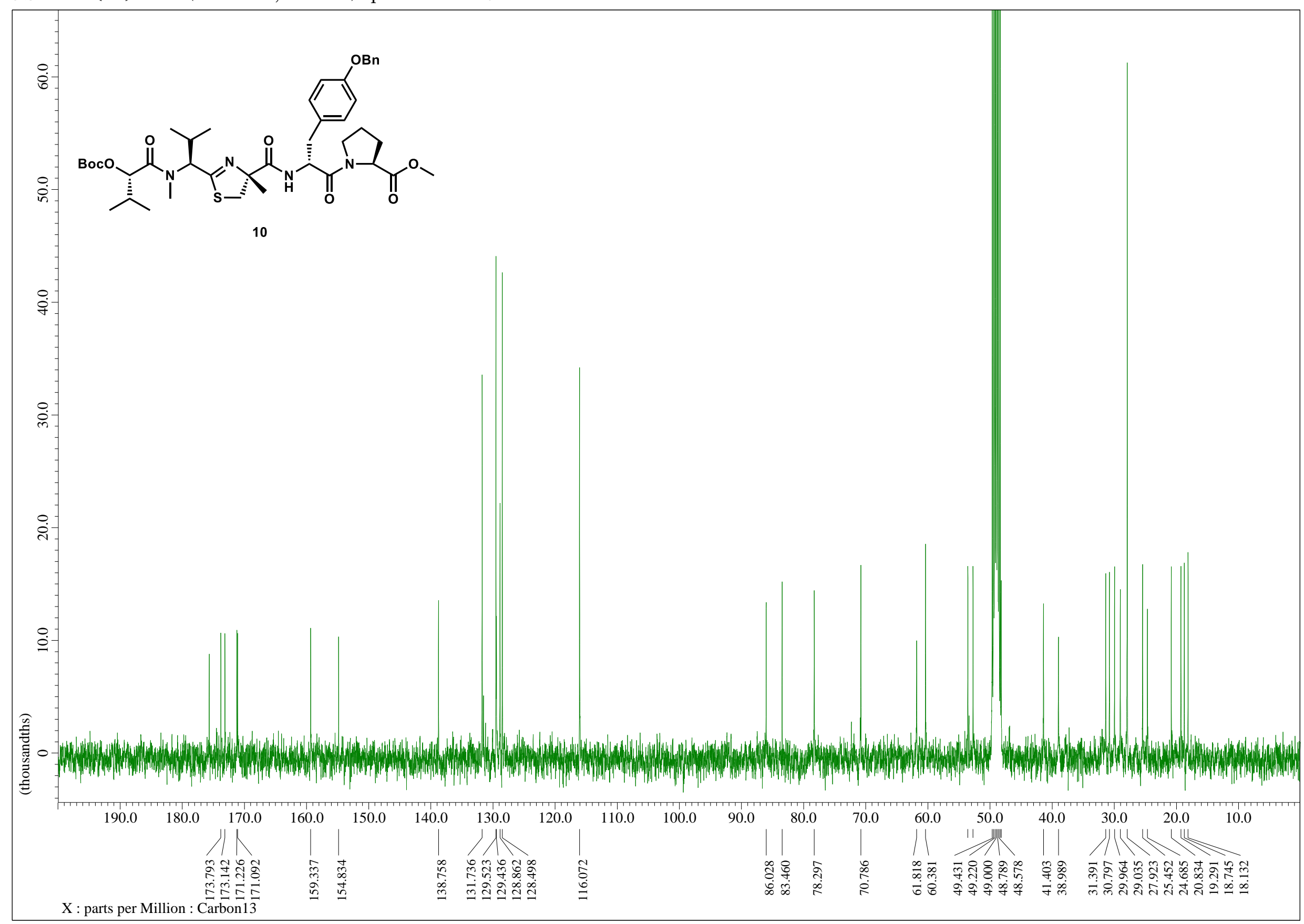


Figure S25. ${ }^{13} \mathrm{C}\{1 \mathrm{H}\}$ NMR (100 MHz, CD $\left.{ }_{3} \mathrm{OD}\right)$ spectrum of 11

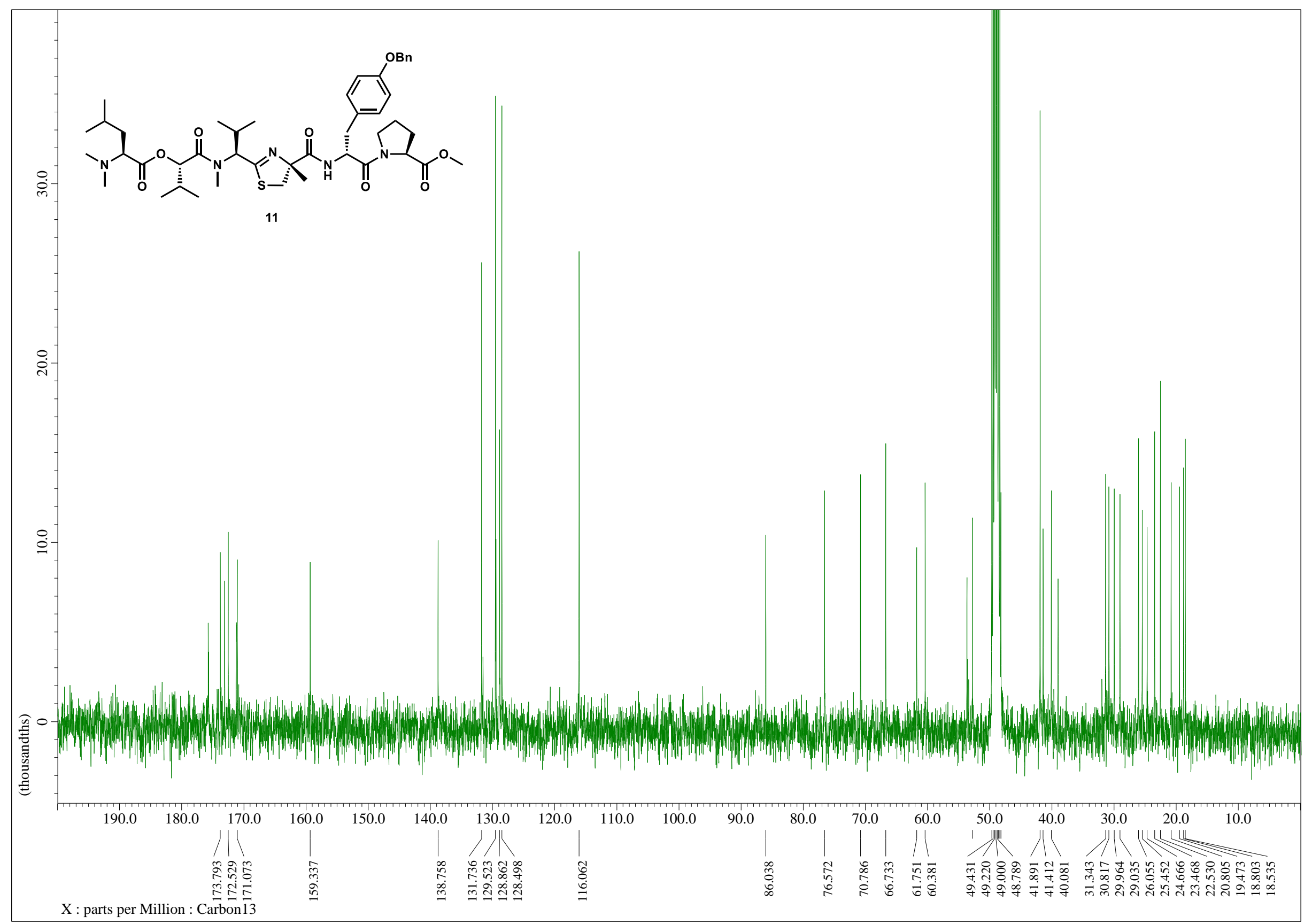


Figure S26. ${ }^{13} \mathrm{C}\{1 \mathrm{H}\} \mathrm{NMR}\left(100 \mathrm{MHz}, \mathrm{CD}_{3} \mathrm{OD}\right)$ spectrum of synthetic kinenzoline (1)

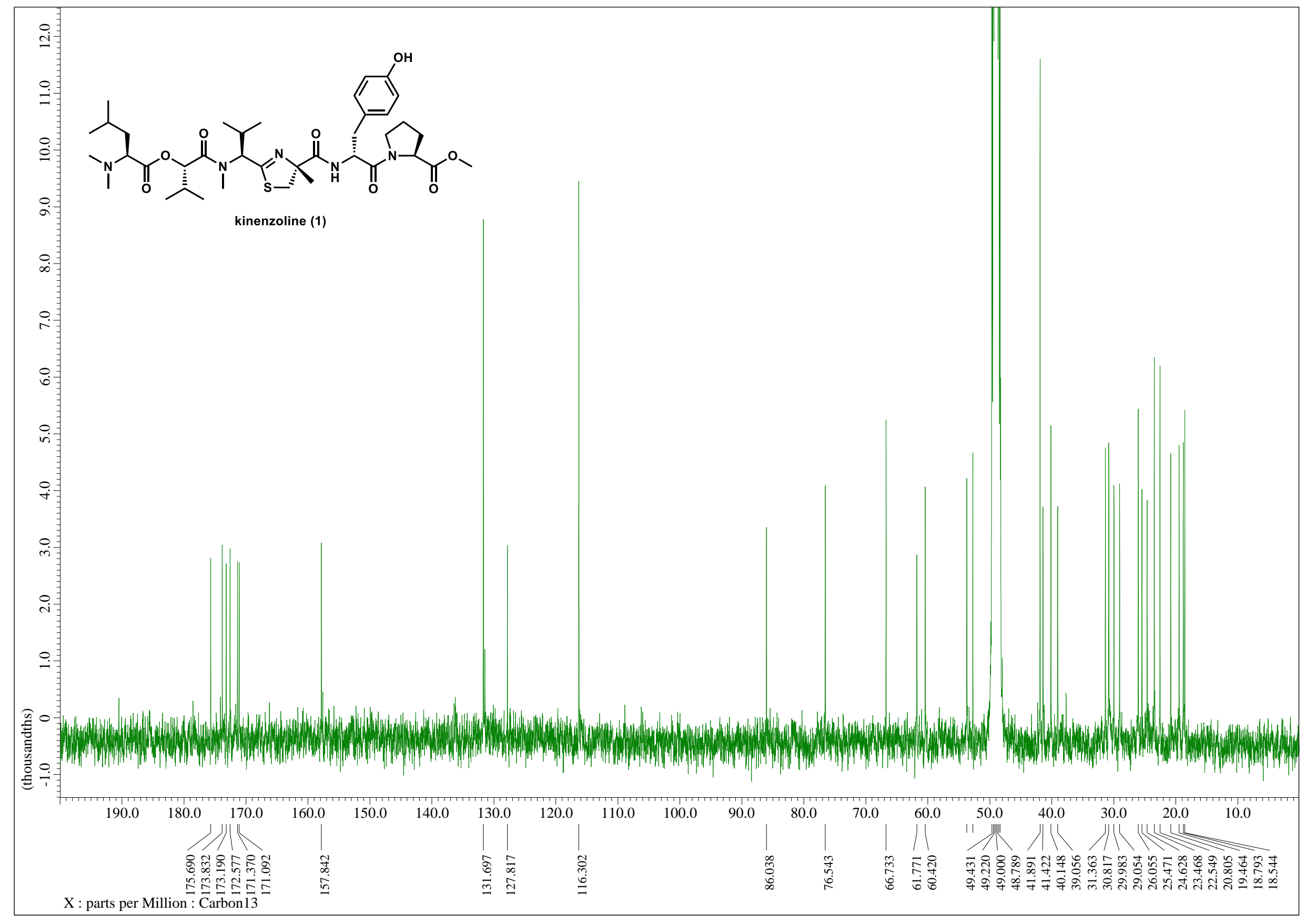

